\title{
REVIEW
}

\section{Hemodynamic Parameters for Cardiovascular System in 4D Flow MRI: Mathematical Definition and Clinical Applications}

\author{
Keiichi Itatani ${ }^{12^{*}}$, Tetsuro Sekine ${ }^{3}$, Masaaki Yamagishi ${ }^{4}$, Yoshinobu Maeda ${ }^{4}$, \\ Norika Higashitani $^{2,5}$, Shohei Miyazaki ${ }^{2}$, Junya Matsuda ${ }^{6}$, and Yasuo Takehara ${ }^{7}$
}

Blood flow imaging becomes an emerging trend in cardiology with the recent progress in computer technology. It not only visualizes colorful flow velocity streamlines but also quantifies the mechanical stress on cardiovascular structures; thus, it can provide the detailed inspections of the pathophysiology of diseases and predict the prognosis of cardiovascular functions. Clinical applications include the comprehensive assessment of hemodynamics and cardiac functions in echocardiography vector flow mapping (VFM), 4D flow MRI, and surgical planning as a simulation medicine in computational fluid dynamics (CFD).

For evaluation of the hemodynamics, novel mathematically derived parameters obtained using measured velocity distributions are essential. Among them, the traditional and typical parameters are wall shear stress (WSS) and its related parameters. These parameters indicate the mechanical damages to endothelial cells, resulting in degenerative intimal change in vascular diseases. Apart from WSS, there are abundant parameters that describe the strength of the vortical and/or helical flow patterns. For instance, vorticity, enstrophy, and circulation indicate the rotating flow strength or power of $2 \mathrm{D}$ vortical flows. In addition, helicity, which is defined as the cross-linking number of the vortex filaments, indicates the 3D helical flow strength and adequately describes the turbulent flow in the aortic root in cases with complicated anatomies. For the description of turbulence caused by the diseased flow, there exist two types of parameters based on completely different concepts, namely: energy loss (EL) and turbulent kinetic energy (TKE). EL is the dissipated energy with blood viscosity and evaluates the cardiac workload related to the prognosis of heart failure. TKE describes the fluctuation in kinetic energy during turbulence, which describes the severity of the diseases that cause jet flow. These parameters are based on intuitive and clear physiological concepts, and are suitable for in vivo flow measurements using inner velocity profiles.

Keywords: blood flow imaging, cardiac surgery, 4D flow magnetic resonance imaging, hemodynamics

'Department of Cardiovascular Surgery, Osaka City University, Osaka, Osaka, Japan

2Cardio Flow Design Inc., Tokyo, Japan

'Department of Radiology, Nippon Medical School Musashi Kosugi Hospital, Kawasaki, Kanagawa, Japan

"Department of Pediatric Cardiovascular Surgery, Kyoto Prefectural University of Medicine, Kyoto, Kyoto, Japan

'Department of Cardiovascular Surgery, Kyoto Prefectural University of Medicine, Kyoto, Kyoto, Japan

'Department of Cardiovascular Medicine, Nippon Medical School, Tokyo, Japan 'Department of Fundamental Development for Advanced Low Invasive Diagnostic Imaging, Nagoya university Graduate School of Medicine, Nagoya, Aichi, Japan

*Corresponding author: Department of Cardiovascular Surgery, Osaka City University, 1-5-7, Asahi-cho, Abeno-ku, Osaka. Osaka 545-8586, Japan. Phone: +81-6-6645-2121, E-mail: keiichiitatani@gmail.com

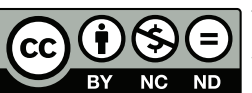

This work is licensed under a Creative Commons Attribution-NonCommercial-NoDerivatives International License.

(C2022 Japanese Society for Magnetic Resonance in Medicine

Received: July 6, 2021 | Accepted: December 22, 2021

\section{Introduction}

Currently, blood flow imaging is one of the emerging trends in cardiology, and the use of technologies, such as 4D flow MRI and computational fluid dynamics (CFD), has been widespread with novel commercial software and services. ${ }^{1,2}$ Because cine mode MRI performs systematic scans of the whole heart to examine its cardiac functions, 4D flow MRI enables comprehensive assessment of cardiac function and hemodynamics when combined with traditional cine mode scanning processes such as steady-state free precession (SSFP) scanning even in patients with complex 3D anatomies. ${ }^{2}$ Because CFD is a technology for mathematical simulation, surgical planning for the selection of the optimal procedure can be achieved when combined with computer graphics $(\mathrm{CG})$. At present, blood flow imaging tools play a key role in clinical applications, in addition to their traditional role as research tool for obtaining clear, colorful pictures and videos of flow streamline pressure 
contours. Therefore, methods to assess the hemodynamics from the visualized blood flow are considered to be advantageous to clinical scientists. Therefore, indices or parameters of hemodynamics would serve as a compass to guide the detailed inspection of the dynamics of the cardiovascular system. The mathematical and/or physical definitions of these parameters are closely linked to the physiological, pathological, and clinical meanings of the diseases because most of them describe either the characteristics of the diseased flow or the mechanical stress in the cardiovascular system. ${ }^{2}$

Typical parameters of visualized blood flow are wall shear stress (WSS), which is defined based on the flow velocity profile near the cardiovascular wall, ${ }^{3-7}$ and its related parameters. There exist many discussions regarding WSS and the intimal damage or degeneration on vessels. A significantly high WSS is known to cause plaque rupture ${ }^{5}$ or intimal damage, ${ }^{7}$ whereas too significantly low WSS is known to cause plaque progression. ${ }^{6}$ The fluctuation in WSS in a cardiac cycle, represented by the oscillatory shear index (OSI), is also known to maintain a comfortable endothelial environment to avoid free radical production. ${ }^{3}$ Moreover, there are several controversial reports on the role of WSS, ${ }^{8}$ in addition to numeral discussions and reviews on WSS and its related parameters based on both in vivo observations ${ }^{8,9}$ and experimental studies. ${ }^{10,11}$ However, because WSS is calculated from the near wall velocity profile, it is highly dependent on the spatial resolution, thereby substantially limiting current MRI machine performances. Therefore, in this review, we focus on the parameters that define the flow pattern inside the cardiovascular lumen rather than near the cardiovascular wall, and those that are considered to be advantageous for in vivo blood flow visualization tools, including 4D flow MRI and echocardiography vector flow mapping (VFM).

\section{Parameter Derivation in 4D Flow MRI and Validation Methods with Experiments for Accuracy, Precision, Reproducibility, and Feasibility}

Most of the parameters derived from measured velocity component distributions in the cardiovascular system obtained from 4D flow MRI are defined by mathematical calculation of the combination of arithmetic and partial differential operations on the velocity vector fields. For this reason, accuracy is considered to be dependent on the resolution and data noise of the MRI measurements. There have been many experimental trials to estimate the error of these parameters because even in a limited accuracy, if precision or reproducibility of these parameters is elucidated, they have been expected to be useful in clinical and practical situations. Because 4D flow MRI is the only method to measure 3D flow velocity fields with full vector components in vivo, most of the previous validation studies are experimental ones including numerical experiments with $\mathrm{CFD}^{12,13}$ or silicon model experiments with optical particle imaging velocimetry (PIV) or particle tracking velocimetry (PTV) ${ }^{14-16}$ CFD can completely eliminate data noise and can even imitate physiological pulsatile flow when the boundary conditions are defined properly, even though it provides only calculated results, not actually measured ones. ${ }^{2,13}$ PIV or PTV can perform in vitro detail experiments that enable comparison of velocity vector components even though the flow would be artificial and a little bit far from physiological ones such as continuous or simplified pulsatile ones. ${ }^{16}$

\section{Parameters to Evaluate Vortical and Helical Flow}

The 3D vortex flow stream has fascinated humans since Leonardo da Vinci painted a picture of the vortex flow in the Valsalva sinus. Kilner et al. first visualized the actual vortex in the sinus with PC-MRI. ${ }^{17}$ Currently, the sophisticated vortical and helical flows inside cardiovascular structures can be visualized with sufficient spatial and temporal resolutions using 4D flow MRI.

For the quantification of the strength of the vortical flow, vorticity, $\vec{\omega}$ with rotational flow direction is defined by the following formula:

$$
\vec{\omega}=\operatorname{rot}(\vec{u})=\nabla \times \vec{u}
$$

where $\vec{u}\left(u_{x}, u_{y}, u_{z}\right)$ describes flow velocity vector in arbitrary time and position. In 3D Euclidean coordinates, vorticity vector $\omega$ components are described as follows:

$$
\vec{\omega}=\left(\frac{\partial u_{z}}{\partial y}-\frac{\partial u_{y}}{\partial z}, \frac{\partial u_{x}}{\partial z}-\frac{\partial u_{z}}{\partial x}, \frac{\partial u_{y}}{\partial x}-\frac{\partial u_{x}}{\partial y}\right)
$$

In a $2 \mathrm{D}$ flow, in which $u_{z}=0$ (constant), $\frac{\partial u_{x}}{\partial z}=0$, and $\frac{\partial u_{y}}{\partial z}=0, \omega$ becomes a scalar quantity (with only the $\mathrm{Z}$ component).

$$
\omega=\frac{\partial u_{y}}{\partial x}-\frac{\partial u_{x}}{\partial y}
$$

In a $2 \mathrm{D}$ vortex, a clockwise vortex exhibits negative vorticity, whereas a counterclockwise vortex exhibits positive vorticity.

Vorticity is a parameter used to evaluate the local swirling flow strength microscopically; however, vorticity does not directly provide information regarding the macroscopic vortex flow. ${ }^{1}$ Circulation is a parameter used to define the rotational speed of a closed loop $C$.

$$
\text { Circulation }=\oint_{C} \vec{u} \cdot \overrightarrow{d l}
$$

If Green's theorem is applied to a region $D$ bounded by a closed loop C, Circulation becomes 


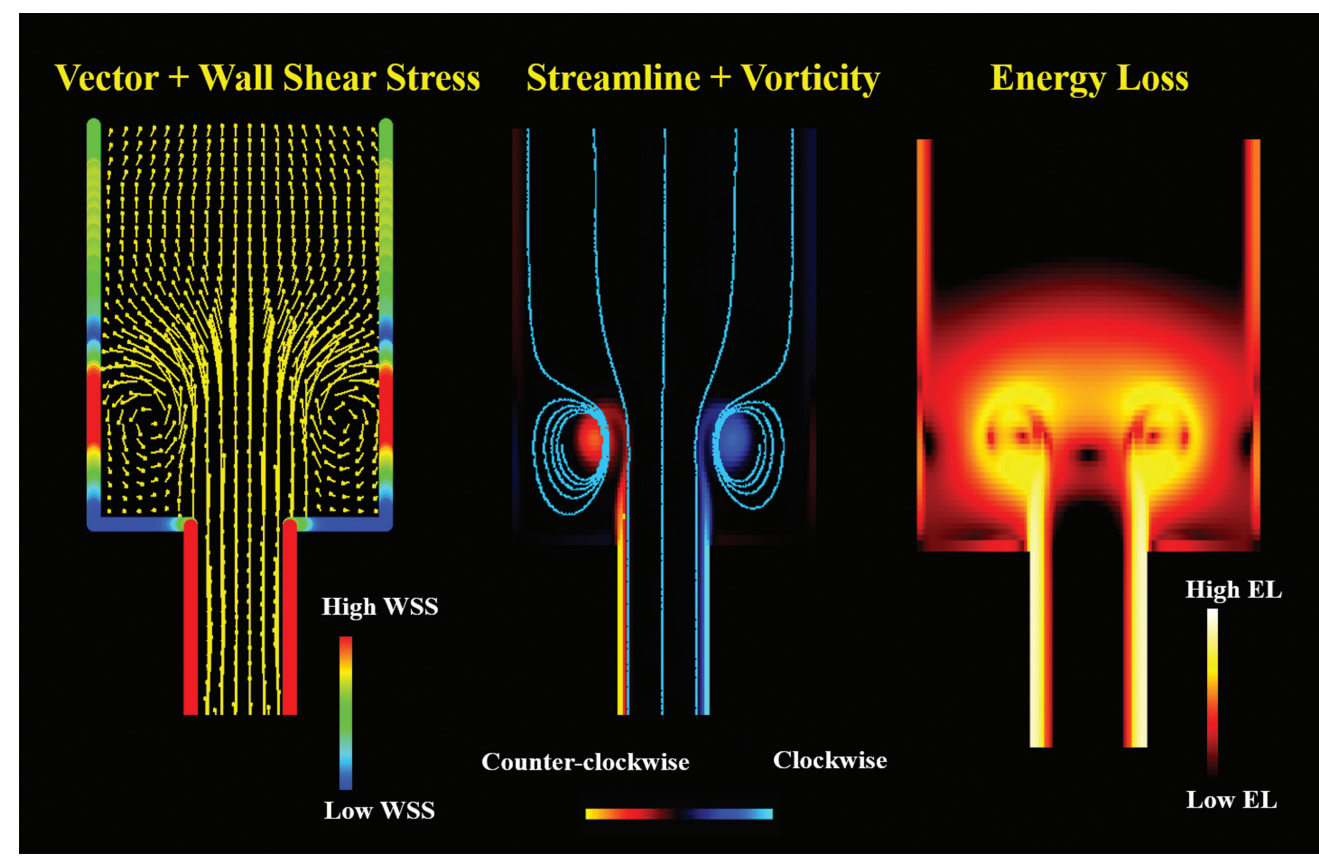

Fig. 1 Conceptual description of flow velocity and streamline with WSS, vorticity, and flow EL. ${ }^{1,18}$ $\mathrm{EL}$, energy loss; WSS, wall shear stress.

$$
\text { Circulation }=\iint_{D} \operatorname{rot}(\vec{u}) \cdot \vec{n} d A=\iint_{D} \vec{\omega} \cdot \vec{n} d A
$$

where $d A$ indicates an incremental area. Thus, the circulation is the surface integral of the vorticity within a region $D$. Vortex flow structures in the human LV are believed to facilitate smooth diastolic filling or smooth ejection toward outflow; thus, swirling flow characteristics are believed to be important underlying mechanisms of ventricular failure. ${ }^{1}$ Figs. 1 and 2 illustrate the concept of vortex flow pattern (Fig. 1) $)^{1,18}$ and its application to the blood flow inside the LV observed in echocardiography VFM (Fig. 2). ${ }^{1,18,19}$

Total swirling strength of a $3 \mathrm{D}$ region $\Omega$ is defined as Enstrophy $S$.

$$
S=\frac{1}{2} \int_{\Omega}|\vec{\omega}|^{2} d v
$$

Enstrophy is a useful parameter used to describe the structure of the turbulence and is often discussed in relation to kinetic energy $(K E)$.

$$
K E=\frac{1}{2} \int_{\Omega}|\vec{u}|^{2} d v
$$

The important difference between $S$ and $K E$ is the local invariance in the mean flow velocity, i.e. $S$ is invariant under the Galilean transformation, whereas $K E$ is dependent on the arbitrary mean field velocity $\mathrm{V}$.

$$
x^{\prime}=x+V t
$$

$$
t^{\prime}=t
$$

Therefore, as a parameter of vortex flow strength, $S$ is invariant with moving coordinates such as that on the moving red blood cell particles with rotating motion. However, a limited number on reports of clinical observations regarding $S$ have been documented thus far, including those on rotational flow in the abdominal aortic aneurysm, ${ }^{20}$ and physiological rotational flow in the right atrium. ${ }^{21}$ A vortex is a type of phenomenon observed in 2D, and the parameters related to the strength of the vortex are often discussed in 2D observation. $^{18,19,22}$

However, the swirling character of $3 \mathrm{D}$ flow is somewhat more complicated than vortex flow because of the helical spiral component of the flow stream in 3D. The parameter to evaluate the helical flow structure is Helicity $H$.

$$
H=\int_{\Omega} \vec{u} \cdot \vec{\omega} d v
$$

$H$ refers to the torsion of the spiral flow stream, whereas $S$ refers to the rotating strength in the defined region. The geometrical image of this parameter represents a cross-linking number of vortex filaments (Fig. 3). ${ }^{1,23}$ For example, when two closed-loop vortex filaments $\mathrm{C} 1$ and $\mathrm{C} 2$ are cross-linked with 


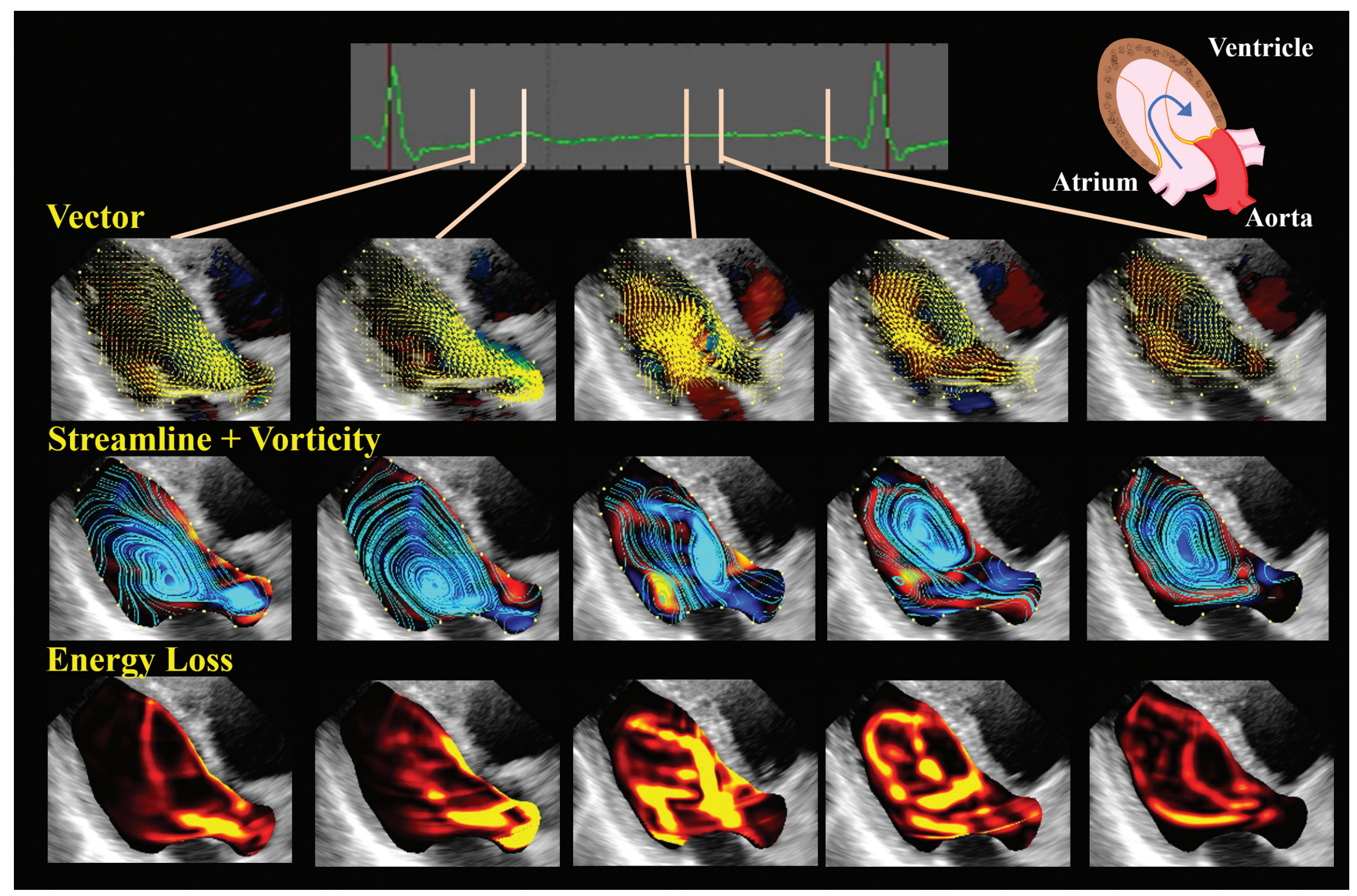

Fig. 2 Normal left ventricular flow pattern with vorticity and flow energy loss. ${ }^{1,19}$

each other, and the cross-sectional vortex filament fluxes are Q1 and $\mathrm{Q} 2$, respectively, then the cross-linking number of the vortex filaments is defined as the number of coverages of a unit spherical surface of the projected unit vector of two points distance along $\mathrm{C} 1$ and $\mathrm{C} 2$ (Fig. 4). ${ }^{1}$

$$
\begin{aligned}
& \text { Cross Linking Number } \operatorname{lk}\left(C_{1}, C_{2}\right) \\
& \quad=\frac{1}{4 \pi} \iint \frac{d \vec{x}}{d t} \times \frac{d \vec{y}}{d s} \cdot \frac{\vec{x}-\vec{y}}{|\vec{x}-\vec{y}|^{3}} d t d s
\end{aligned}
$$

With the flux of vorticities Q1 and Q2, we have

$$
\begin{aligned}
& \frac{1}{4 \pi} \iint \frac{d \vec{x}}{d t} \times \frac{d \vec{y}}{d s} \cdot \frac{\vec{x}-\vec{y}}{|\vec{x}-\vec{y}|^{3}} d t d s \int \overrightarrow{\omega(x)} d A(x) \int \overrightarrow{\omega(y)} d A(y) \\
& \quad=\frac{1}{4 \pi} \int \overrightarrow{\omega(x)} d v_{x} \cdot \int \overrightarrow{\omega(y)} \times \frac{\vec{x}-\vec{y}}{|\vec{x}-\vec{y}|^{3}} d v_{y} \\
& \quad=\frac{1}{4 \pi} \int \overrightarrow{\omega(x)} d v_{x} \cdot \operatorname{rot}^{-1}(\vec{\omega})=\int \vec{\omega} \cdot \vec{u} d v=H
\end{aligned}
$$

under the well-known law of Biot-Savart.

$$
\frac{1}{4 \pi} \int \overrightarrow{\omega(y)} \times \frac{\vec{x}-\vec{y}}{|\vec{x}-\vec{y}|^{3}} d y \omega_{y}=\operatorname{rot}^{-1}(\vec{\omega})=\vec{u}
$$

Alternatively, under the theorem of Stokes,

$$
\begin{aligned}
\int_{i^{\text {th }} \text { tube }} \vec{u} \cdot \vec{\omega} d A_{1} d C_{i} & =\int_{C_{i}} \int_{A_{1}} \vec{u} \cdot\left(\vec{\omega} d A_{1}\right) d C_{i} \\
& =Q_{1} \int_{C_{i}} \vec{u} d C_{i} \\
& =Q_{1} l k\left(C_{1}, C_{i}\right) Q_{i} \\
& =Q_{1} l k\left(C_{1}, C_{i}\right) \int_{A_{i}} \operatorname{rot}(\vec{u}) d A_{i} \\
& =Q_{1} l k\left(C_{1}, C_{i}\right) Q_{i}
\end{aligned}
$$

Thus,

$$
\begin{aligned}
H & =\int \vec{u} \cdot \vec{\omega} d v=\frac{1}{2}\left\{Q_{1} \int_{C_{1}} \vec{u} d C_{1}+Q_{2} \int_{C_{2}} \vec{u} d C_{2}\right\} \\
& =Q_{1} Q_{2} l k\left(C_{1}, C_{2}\right)
\end{aligned}
$$



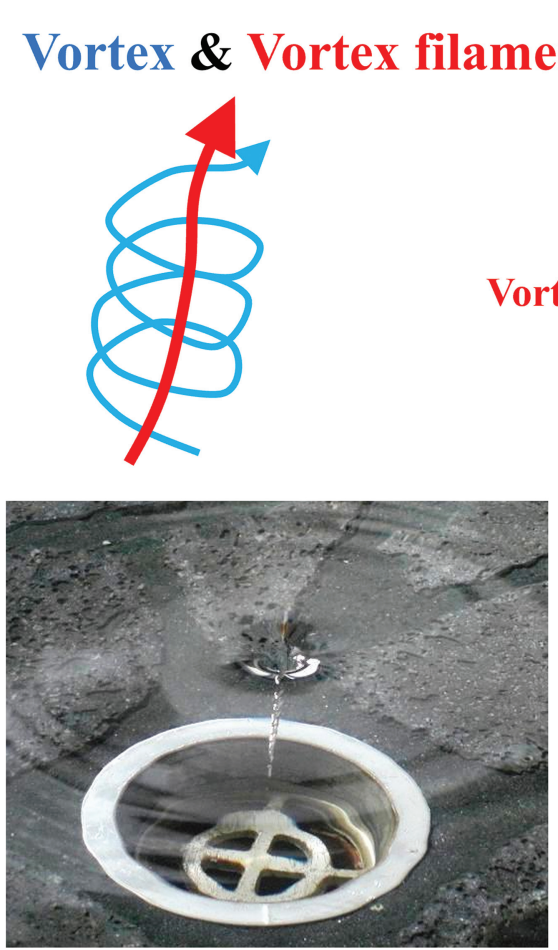

Vortex filament

\section{Well-aligned swirling flow: Low helicity flow}

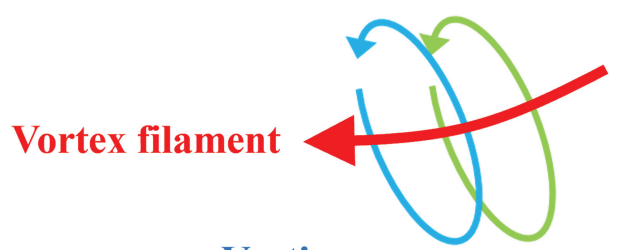

Vortices

\section{Disturbed swirling flow: High helicity flow}

Vortex filaments

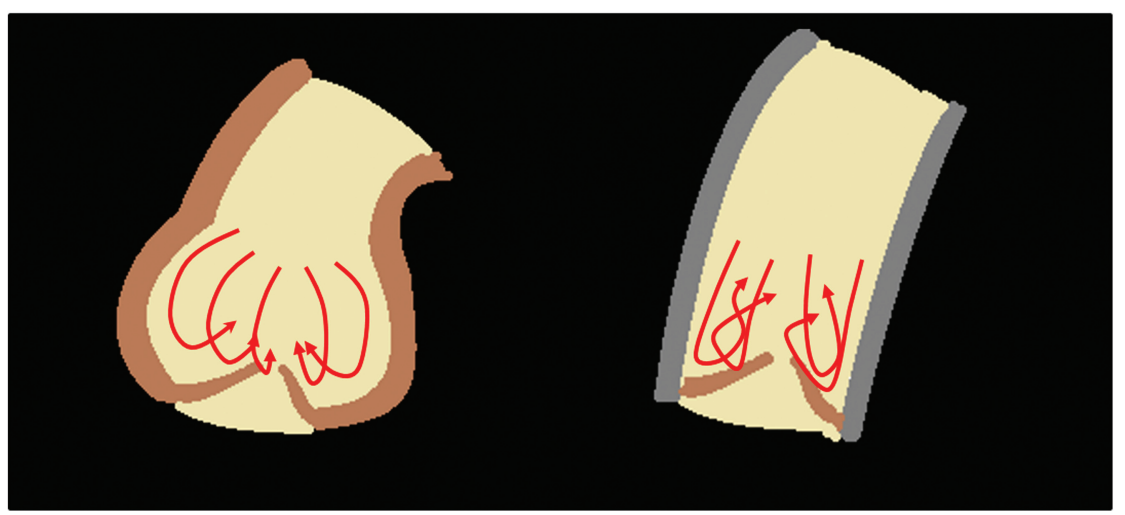

Fig. 3 Vortex filament and concept of helicity. ${ }^{1}$ Vortex filament is a kind of central core of the vortical flow defined by the high vorticity region of the local vortical flow (the left). In the cardiovascular system, vortex flow is often observed, but in many situations of the natural anatomy, the well-aligned vortex flow with low helicity without linking of the vortex filaments should be observed (the middle), but after the non-anatomical reconstruction or diseased state, disturbed malaligned flow with high helicity with linking of the vortex filaments is assumed to be observed (the right).

From the viewpoint of dissipation of physical quantities, in addition to $K E$ and $S, H$ can also be considered as a conservative quantity that dissipates with viscosity. The difference between vortical and helical flow streamlines is often clear in aortic valve diseases, where the bicuspid aortic valve causes high helicity flow inside the ascending aorta. ${ }^{24,25}$ After aortic valve surgery, a porcine aortic bioprosthetic valve tends to exhibit spiral helical flow, whereas bovine pericardial bioprosthesis tends to cause vortical flow inside the ascending aorta owing to the difference in valve structures. ${ }^{26}$ Flow pattern observation of these helical and vortical flows is one of the optimal applications of $4 \mathrm{D}$ flow MRI ${ }^{27}$ thus, detailed experimental validations of these parameters have been performed. ${ }^{28}$ Helicity and vorticity have been measured in a wide range of cardiovascular diseases, including pulmonary arterial hypertension cases, ${ }^{29}$ aortic diseases such as aortic dissection ${ }^{30}$ coarctation of aorta, ${ }^{31}$ and even arterio-venous fistula in hemodialysis patients, ${ }^{32}$ as well as reference to healthy normal patterns, ${ }^{33}$ their changes with advancing age have also been measured. ${ }^{34}$

Because these parameters are derived from a one-time spatial derivative of the velocity vector field, their accuracy would be expected to be dependent on the resolution of the voxels. However, even in a complicated vortical or helical flow caused by the flow collision at the Fontan anastomosis site, vorticity and helicity were reported to capture the essential characteristics of the complicated vortical or helical flow both in 4D flow MRI and in optical PIV, in in vitro experiments of continuous and pulsatile flows. ${ }^{16}$

Because the aortic flow is affected not only by the aortic valve but also by the anatomy of the aortic root, helicity is one of the comprehensive and indicative parameters to evaluate the extent of inefficiency in blood flow inside the aortic root. Adult congenital heart disease (ACHD) patients often have dilated aortic roots with anatomical malalignment of the root structure, and helical spiral flow is reported to have been observed in the aortic root, such as the overriding of the aortic root in the tetralogy of Fallot. ${ }^{35}$ Fig. 5 (unpublished data) describes ACHD samples with highly cross-linked vortex filaments in the aortic root compared with the healthy normal spiral aortic root flow pattern with a well-aligned vortex filaments with small Helicity. In complicated heart anatomy, blood flow stream sometimes shows complicated helical flow with heavy cross-link. These heavily cross-linked vortex filaments with high Helicity induce high mechanical stress locally 

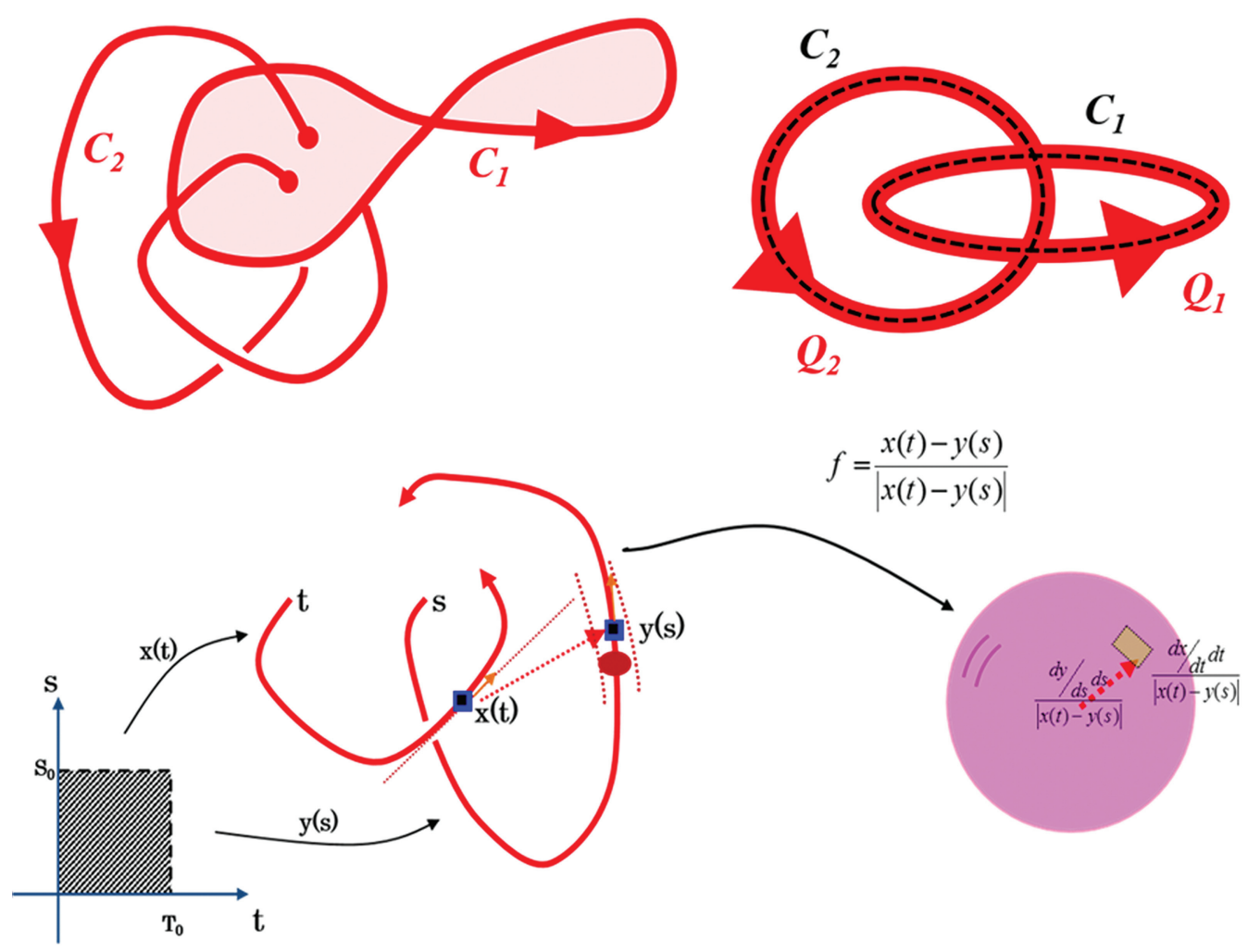

Fig. 4 Cross-linking number of vortex filaments and its mathematical definition. ${ }^{1}$ Mathematical definition of the cross-linking number of the two closed loop should be the number along a loop passing the surface defined by the other loop (the upper), or should be the coverage number of a sphere defined by the unit vector from the two arbitrary points of each loop, which can be defined as a map from a square in a 2D Euclidean plane of two parameters (the lower).

on the aortic valve leaflets; therefore, reduced helical flow patterns with well-aligned root flow streams are desired in aortic valve surgery. ${ }^{26,36}$ Hence, kinetic energy, Enstrophy, and Helicity are the types of parameters to be dissipated with viscosity.

\section{Parameter to evaluate flow energy loss cause and cardiac workload}

Although parameters that describe the vortical or helical flow patterns can evaluate the local flow structures quantitatively, a global parameter to estimate unfavorable flow structure is required when physicians consider the pathophysiology of diseases. Therefore, energy loss (EL) has been defined as the drops in energy level from the inlets to the outlets.

$$
E L=\sum_{\text {Inlet }}\left(\frac{1}{2} \rho|\vec{u}|^{2}+P\right) Q-\sum_{\text {outlet }}\left(\frac{1}{2} \rho|\vec{u}|^{2}+P\right) Q
$$

where $\rho$ is density of the blood and $P$ is the pressure, and total pressure (TP) is defined as follows:

$$
T P=\frac{1}{2} \rho|\vec{u}|^{2}+P
$$

The first term refers to kinetic pressure, the second term refers to static pressure, and TP represents the total energetic potential of the blood flow. At the stenosis site, kinetic pressure increases with flow acceleration, static pressure decreases, and the total pressure remains constant; however, with flow deceleration distal to the stenosis site, the kinetic pressure decreases substantially, and static pressure recovers to some extent. This phenomenon is termed as pressure recovery and the total pressure decreases according to the amount of energy lost by the inefficient jet flow or vortex flow distal to the site of stenosis. The EL in this form considered as an important parameter to estimate the cardiac workload in cardiac diseases. The EL coefficient, or corrected effective orifice area in the assessment of aortic stenosis (AS), that was first derived by Garcia et al. in 2000 includes the concept of pressure recovery to diagnose the severity of $\mathrm{AS},{ }^{37}$ achieving reasonably accurate prediction of AS patients' long-term prognosis. ${ }^{38}$ The EL defined with the TP drop as a factor has played an important role in the progressive surgical design of the Fontan procedure including the comparison between the different types of Fontan 


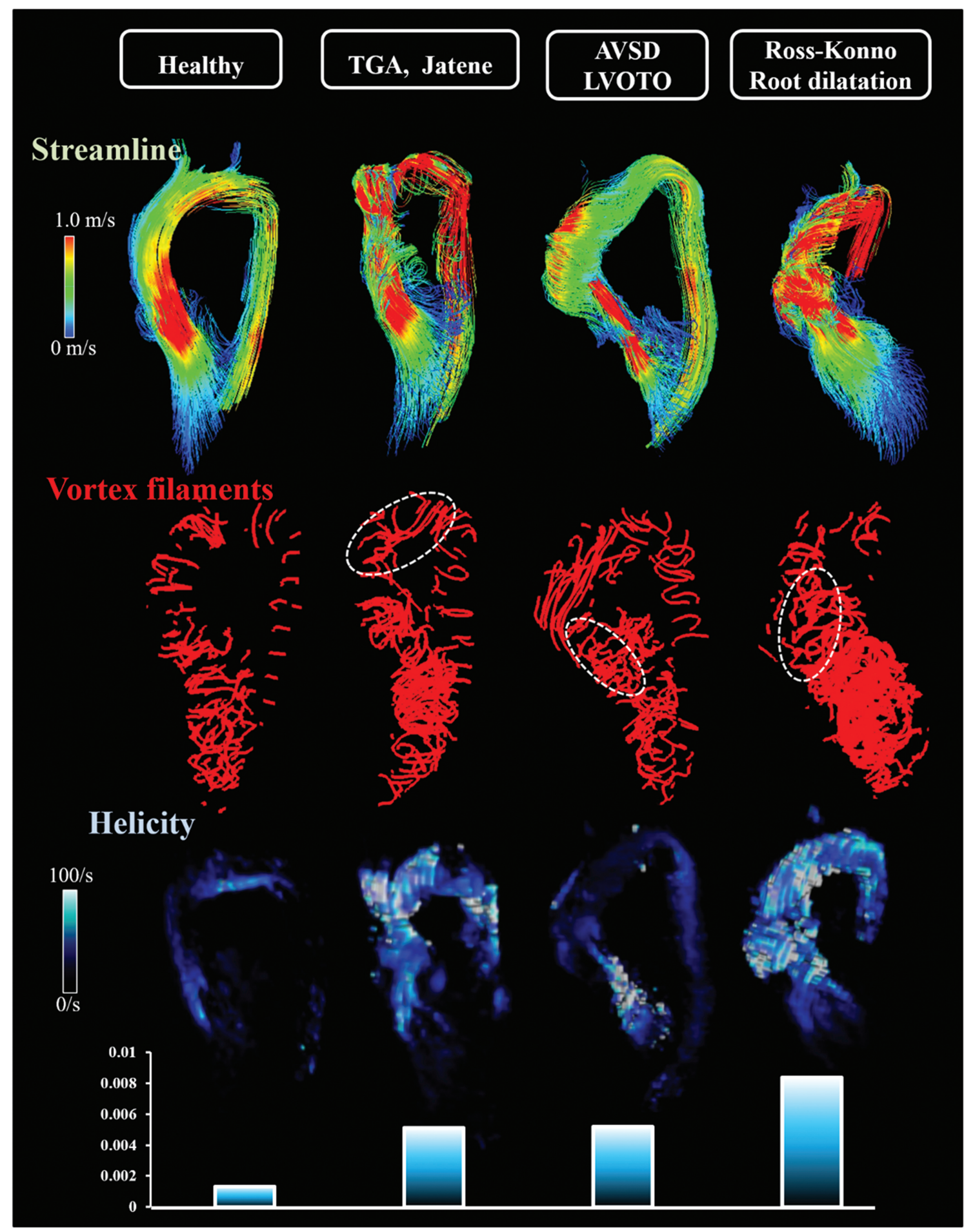

Fig. 5 Sample of helicity and cross-linking of vortex filaments. In the case of a 29-year-old man with arterial switch operation (Jatene procedure) in the TGA, the LVOT was distorted to the ascending aorta, and the Valsalva sinus was slightly dilated. In the case of a 59-year-old man with LVOTO in the AVSD repair and mitral annuloplasty with an artificial ring, the LVOT jet in a slightly elongated aortic root caused highly spiral helical flow with heavily cross-linked vortex filaments. In the case of a 31 -yearold man with prominent root dilatation with severe aortic insufficiency after the Ross procedure, chaotically confused vortex filaments with prominently high Helicity were observed. Distorted LVOT, jet flow and dilated aortic root causes highly crosslinked vortex filaments, resulting in an increase in helicity (Unpublished data). AVSD: atrioventricular septal defect; LVOTO: left ventricular outflow tract obstruction; TGA: transposition of the great arteries. 
procedures, ${ }^{39-42}$ geometrical configurations, including the anastomosis site of the total cavo-pulmonary connections, ${ }^{43}$ and the size of the extracardiac conduit, ${ }^{44,45}$ the effect of pulmonary arterial vasculature unbalance or stenosis ${ }^{46-48}$ under the anatomical variance, ${ }^{49}$ and the effect of exercise, ${ }^{45,48,50,51}$ based on CFD, because even a small amount of EL is susceptible to single ventricular physiology as a cardiac workload. In 2011, Itatani et al. derived a novel method of calculating EL without pressure information. ${ }^{52}$ Thus, the 4D flow MRI, VFM, and other blood flow visualization tools enabled the quantification and visualization of EL when the blood flow velocity distribution data are available. ${ }^{18,52,53}$ Because this parameter is mathematically derived as shown in the following equations, the accuracy of EL as an energy level drop should be conceptually proven, but only the method of experimental validation can be performed numerically because the derivation process includes pressure term even though the definition of EL eliminates the pressure, and this conceptual proof has already been performed with numerical experiments. ${ }^{52,53}$

Changes in energy can be expressed as follows:

$$
d\left(\frac{1}{2} \rho|\vec{u}|^{2}+e\right)=d\left(\frac{1}{2} \rho|\vec{u}|^{2}\right)+\frac{T d S-P d V}{V}
$$

where $\rho, P, T, S, e$, and $V$ indicate density, pressure, temperature, entropy, internal energy, and volume, respectively. The right-hand side of equation (18) is derived from the first and second laws of thermodynamics. In an incompressible and adiabatic system, only the first term should be examined.

In the case of blood flow, an incompressible fluid, the mass, and momentum preservation equations are expressed as follows:

$$
\begin{gathered}
\nabla \cdot \vec{u}=0 \\
\rho \frac{\partial \vec{u}}{\partial t}=-\rho(\vec{u} \cdot \nabla) \vec{u}-\nabla P+\mu \Delta \vec{u}
\end{gathered}
$$

where $\mu$ denotes the viscosity. Equation (19) is a continuity equation and equation (20) is the Navier-Stokes equation.

When the time derivative of the energy is substituted in equations (19) and (20), we obtain

$$
\begin{aligned}
\frac{\partial}{\partial t}\left(\frac{1}{2} \rho|\vec{u}|^{2}\right) & =-\rho \vec{u} \cdot(\vec{u} \cdot \nabla) \vec{u}-\vec{u} \cdot \nabla P+\sum_{i, j} u_{i} \frac{\partial 2 \sigma_{i, j}}{\partial x_{j}} \\
& =-(\vec{u} \cdot \nabla)\left(\frac{1}{2} \rho|\vec{u}|^{2}+P\right)+\nabla \cdot\left(\sum_{i} u_{i} \cdot 2 \sigma_{i, j}\right) \quad ; \text { then, } \\
& -\sum_{i, j} 2 \sigma_{i, j} \frac{\partial u_{i}}{\partial x_{j}}
\end{aligned}
$$

where the tensor $\sigma_{i, j}$ is a shear stress tensor, defined as

$$
\sigma_{i, j}=\frac{1}{2} \mu\left(\frac{\partial u_{j}}{\partial x_{i}}+\frac{\partial u_{i}}{\partial x_{j}}\right)
$$

Because blood is incompressible, continuity equation (19) can be applied to equation (21), and the first term on the right-hand side of the equation can be defined as a divergence:

$$
\begin{aligned}
\frac{\partial}{\partial t}\left(\frac{1}{2} \rho|\vec{u}|^{2}\right)= & -\nabla \cdot\left(\left(\frac{1}{2} \rho|\vec{u}|^{2}+P\right) \vec{u}-\sum_{i} 2 u_{i} \cdot \sigma_{i, j}\right) \\
& -\sum_{i, j} 2 \sigma_{i, j} \frac{\partial u_{i}}{\partial x_{j}}
\end{aligned}
$$

When equation (23) is integrated over the whole volume of concern, such as the site of vessel anastomosis, and when the space integral and time derivative are exchanged with the Gauss theorem and the continuity equation (19), respectively, the following equation is obtained:

$$
\begin{aligned}
\frac{\partial}{\partial t}\left(\int \frac{1}{2} \rho|\vec{u}|^{2} d v\right)= & -\int\left(\frac{1}{2} \rho|\vec{u}|^{2}+P\right) \vec{u} \cdot \vec{n} d A \\
& +\int\left(\sigma_{i, j} \cdot \vec{u}\right) \cdot \vec{n} d A \\
& -\sum_{i, j} \int \frac{1}{2} \mu\left(\frac{\partial u_{i}}{\partial x_{j}}+\frac{\partial u_{j}}{\partial x_{i}}\right)^{2} d v
\end{aligned}
$$

where $\vec{n}$ is the vector normal to the surface (outward from the domain) $A$ is the section of the surface area, and its integral is for the area.

When we apply a physiological boundary condition assuming blood vessels,

$$
\vec{u}=0(@ \text { vessel wall })
$$

$$
\vec{u} \cdot \vec{n}(@ \text { inlets and outlets })
$$

$$
\frac{\partial u_{i}}{\partial n_{i}}=0(@ \text { inlets and outlets })
$$

$$
\int\left(\sigma_{i, j} \cdot \vec{u}\right) \cdot \vec{n} d A=0
$$


Thus, the integrated energetic changes can be described as follows:

$$
\begin{array}{r}
\frac{\partial}{\partial t} \int\left(\frac{1}{2} \rho|\vec{u}|^{2}\right) d v=-\int\left(\frac{1}{2} \rho|\vec{u}|^{2}+P\right) \vec{u} \cdot \vec{n} d S \\
-\sum_{i, j} \int \frac{1}{2} \mu\left(\frac{\partial u_{i}}{\partial x_{j}}+\frac{\partial u_{j}}{\partial x_{i}}\right)^{2} d v c r
\end{array}
$$

Assuming the existence and uniqueness of the solution of the Navier-Stokes equation and that the pulsatile fluid flow repeats the same fluid field in every cardiac cycle, the following equation can be derived by integrating equation (29) for one cardiac cycle:

$$
\int_{0}^{T} d t \int \frac{1}{2} \rho|\vec{u}|^{2} d v=\left[\int \frac{1}{2} \rho|\vec{u}|^{2} d v\right]_{t=T}-\left[\int \frac{1}{2} \rho|\vec{u}|^{2} d v\right]_{t=0}=0
$$

From equations (15) and (16),

$$
-\int d t \int\left(\frac{1}{2} \rho|\vec{u}|^{2}+P\right) \vec{u} \cdot \vec{n}=\int d t \sum_{i, j} \int \frac{1}{2} \mu\left(\frac{\partial u_{i}}{\partial x_{j}}+\frac{\partial u_{j}}{\partial x_{i}}\right)^{2} d v
$$

The left-hand side of equation (31) represents the EL calculated based on the concept of total pressure, and the righthand side of equation (31) represents the energy dissipation caused by the confliction of the viscous fluid. Therefore, the definition of EL:

$$
E L=\int d t \sum_{i, j} \int \frac{1}{2} \mu\left(\frac{\partial u_{i}}{\partial x_{j}}+\frac{\partial u_{j}}{\partial x_{i}}\right)^{2} d v
$$

requires only the flow velocity distribution and does not include a pressure term; thus, it is applicable to non-invasive flow visualization methods using echocardiography and MRI.

This type of EL was first applied to echocardiography $\mathrm{VFM}^{18}$ in congenital heart diseases, ${ }^{54-57}$ heart valve diseases, ${ }^{58}$ and heart failure ${ }^{59}$ with the establishment of a reference value, ${ }^{55,60}$ subsequently, it was applied to intraoperative evaluation with trans-esophageal echocardiography. ${ }^{60-62}$ This concept of EL was then applied to PCMRI. ${ }^{63}$ In the case of $4 \mathrm{D}$ flow MRI, this parameter is susceptible to the spatial resolution because of the partial differential in space. Miyazaki et al. validated this parameter in comparison with the numerical calculation data of $\mathrm{CFD},{ }^{13}$ and the result demonstrated sufficient and statistically significant correlation between EL calculated in CFD and that in 4D flow MRI, but the underestimation of the parameter in
4D flow MRI due to underestimation of spatial differential of the flow velocity vector components was caused by the low spatial resolution in 4D flow MRI. However, the precision has been reported to be optimal, ${ }^{13}$ and clinical applications with acceptable reproducibility have also been reported from the derivation process of reference value in healthy volunteers. ${ }^{64}$ This parameter is defined with the integral of the volume of the extracted cardiovascular lumen; thus, before the estimation of EL, it must be determined in which part of the cardiovascular system the workload is assumed to be increased in cardiovascular diseases. Of course, too large velocity encoding (VENC) would underestimate EL in slow velocity regions, de-aliasing by the phase correction of over-VENC region with fixed VENC from 1.5 to $2.0 \mathrm{~m} / \mathrm{s}$ would be recommended for the estimation of EL. ${ }^{64}$ In addition, intra-observer or inter-observer differences would be important for the establishment of the reproducibility of this parameter. For EL in echocardiography VFM, Kakizaki et al. performed repetitive evaluation of a single case with different observers and explained the reproducibility inside a single case with different or with the same conditions, ${ }^{59}$ and for EL in 4D flow MRI, Nakaji et al. briefly described the acceptable differences inside intra-observer and demonstrated limited differences between the breathing and breath-hold scan conditions. ${ }^{64}$

In 2014, Barker et al. first proposed viscous energy loss in abdominal aortic flow as a parameter similar to EL for 4D flow MRI, but with a somewhat different definition from $\mathrm{EL}^{65}$

$$
\text { viscous } E L=\sum_{i, j} \int \frac{1}{2} \mu\left(\frac{\partial u_{i}}{\partial x_{j}}+\frac{\partial u_{j}}{\partial x_{i}}-\frac{2}{3}(\nabla \cdot \vec{u}) \delta_{i, j}\right)^{2} d v
$$

with a concept of the viscous dissipation function,

$$
\Phi_{u}=\frac{1}{2} \sum_{i} \sum_{j}\left[\left(\frac{\partial u_{i}}{\partial x_{j}}+\frac{\partial u_{j}}{\partial x_{i}}\right)-\frac{2}{3}(\nabla \cdot \vec{u}) \delta_{i, j}\right]^{2}
$$

where $\delta_{i, j}$ is Kronecker delta and has a value 1 when $I=j$, and 0 when $i \neq j$. This parameter has been applied to a wide range of cardiac diseases, including aortic valve disease ${ }^{66}$ congenital heart disease, ${ }^{67,68}$ right ventricular (RV) flow, ${ }^{69}$ tricuspid surgery, ${ }^{70}$ aortic disease, ${ }^{71,72}$ and their comparison with healthy ${ }^{73}$ or physiological flows. ${ }^{74}$ Surgical application of this viscous $\mathrm{EL}^{75}$ using 4D flow MRI has also been reported confirming the concept, which has already been confirmed in $\mathrm{CFD},{ }^{76-79}$ that a smoothly tapered aortic arch reconstruction in the Norwood procedure for congenital hypoplastic left heart syndrome has hemodynamical advantages. However, the final term in equation (33) should be under the continuity equation of an incompressible fluid, and not a compressible gas, because blood is an incompressible liquid. Therefore, mathematically speaking, viscous EL is essentially equal to EL in 
equation (32). In 4D flow MRI, the continuity equation (19) does not always hold, due to the measurement error. In particular, the error is considered to be high in turbulent flows with high energy. The validation study of this parameter based on PTV has been reported to demonstrate high dependency on spatial resolution, resulting in underestimation of maximum over $80 \%,{ }^{15}$ which is considered to be too high compared with EL in equation (32). Although these two types of EL differ only in additional terms made of continuity equation (19), which should be zero in mathematics, in actual measurements such as 4D flow MRI and optical PIV or PVT, continuity equation do not always be zero due to the measurement errors, which would be considered to cause exaggeration of underestimation in viscous EL.

According to Itatani, who first proposed the concept of EL in cardiac function, ${ }^{19}$ diseased turbulent flow with nonuniform flow velocity vector distribution causes the EL to increase. The increased energy is compensated by the ventricular work and the EL would represent the cardiac workload. Thus, the EL is a comprehensive parameter of workload including systolic afterload and diastolic preload in heart valve diseases. ${ }^{19,52}$ When a heart falls into a heart failure state, where the generated energy is considerably low, the EL would be low, but the EL/generated energy ratio would be high.

This conceptual description of EL is clearly described, especially in RV heart failure in pulmonary valve diseases. We have illustrated the hypothesis regarding the clinical contribution of the EL estimation. ${ }^{19}$ In the normal state, the ventricle efficiently generates a large energy, resulting in low EL. However, when inefficient unphysiological flow occurs owing to cardiovascular disease, the cardiac workload increases as the EL increases; however, the heart function is preserved for a while, to compensate for the workload. When the workload becomes too large, the ventricle decompensates resulting in heart failure, and the EL decreases because the ventricle cannot generate sufficient energy. Diagnostic parameters routinely obtained in our clinical practice mainly measure the ventricular energy generation function; however, practically, a parameter that estimates the cardiac workload is more desirable so as to avoid missing the optimal timing for therapeutic interventions in cardiovascular diseases. ${ }^{19}$ Therefore, EL is an independent parameter from current heart failure state or cardiac remodeling state such as chamber size or ventricular wall motion; instead, it is expected to be a predictor of ventricular deterioration of highly burdened state caused by the cardiac diseases. ${ }^{57,59,63}$

Because the pulmonary circulation system is based on low pressure, the contribution of kinetic energy is large, and EL plays an essential role in the comprehensive assessment of hemodynamics and cardiac workload. Fig. 6 (unpublished data) illustrates the systolic afterload increase in the main pulmonary artery in pulmonary stenosis (PS), diastolic preload increase in the RV with flow collision of tricuspid inflow and pulmonary valve regurgitant flow, and both during pulmonary stenosis with regurgitation (PSR). In ACHD, one of the most common types of long-term diseases is pulmonary valve disease after the intracardiac repair in tetralogy of Fallot and its related diseases including double-outlet RV with pulmonary stenosis pulmonary atresia with ventricular septal defect. Indication of surgical intervention of the pulmonary valve diseases in ACHD is still a challenging problem. ${ }^{80}$ Because of complicated anatomy after congenital heart surgery, and in addition, different from pathophysiology in childhood, ACHD patients face problems in cardiac function due to the ventricular deterioration. While the RV hemodynamics are significantly complex, the RV volume and function in PR are not the sole predictors of RV prognosis. ${ }^{81}$ Often, patients suffer from PSR because of the degenerated valve structure in a narrow RV outflow tract (RVOT) reconstructed with artificial material during childhood. Therefore, for the concomitant assessment of hemodynamics and cardiac function, 4D flow MRI is considered as a promising tool for RV function and pulmonary valve disease. ${ }^{82,83}$

Significant reduction in EL during both the systole and the diastole was observed in the postoperative state with a sufficiently smooth flow streamline without any flow acceleration or regurgitation (Fig. 7, unpublished data) in a bioprosthetic valve replacement case and in a RVOT reconstruction (RVOTR) case with a valved conduit. Owing to the relaxation of flow acceleration by the PS or RVOT stenosis that is released, the systolic afterload is reduced with the decrease in EL in the systole, whereas by the regulation of regurgitation, the diastolic preload is also reduced with the decrease in EL in diastole. RVOTR with expanded polytetrafluoroethylene (ePTFE) conduits with bulging sinuses and tricuspid fan-shaped valves ${ }^{84}$ with a diameter of 22 or $24 \mathrm{~mm}$ was performed for patients under the age of 25 years, and in cases where the posterior wall of the main pulmonary artery (PA) and the valve annulus could not be preserved. Other cases represented PVR with a bioprosthetic valve and annuloplasty using a bovine pericardial patch to place large bioprosthetic valves $(25$ or $27 \mathrm{~mm})$. After surgery, a significantly reduction in the RV endodiastolic and endosysolic volume index (EDVI/ESVI) and an improvement in the RV ejection fraction (EF) were observed (Fig. 8, unpublished data). From the reference value in the healthy RV cases, ${ }^{64}$ the estimated cutoff values of the RV volume and function at which normalization from the data listed in Fig. 8 could be expected are supposed to be quite stringent when compared with those described in the guideline, ${ }^{80}$ as suggested by clinical evidence. ${ }^{81}$

The EL plot with cardiac output (CO) graphically illustrates the concept of EL quite well (Fig. 9). Regarding PS, energy loss index (ELI) increases with the increase in $\mathrm{CI}$, and in comparison with the normal $\mathrm{RV}$ and postoperative state, the slope of ELI over CI, or the EL/CO ratio is significantly higher in the isolated PS $(\mathrm{n}=15)$ group (Fig. 9b). Regarding the regurgitation 


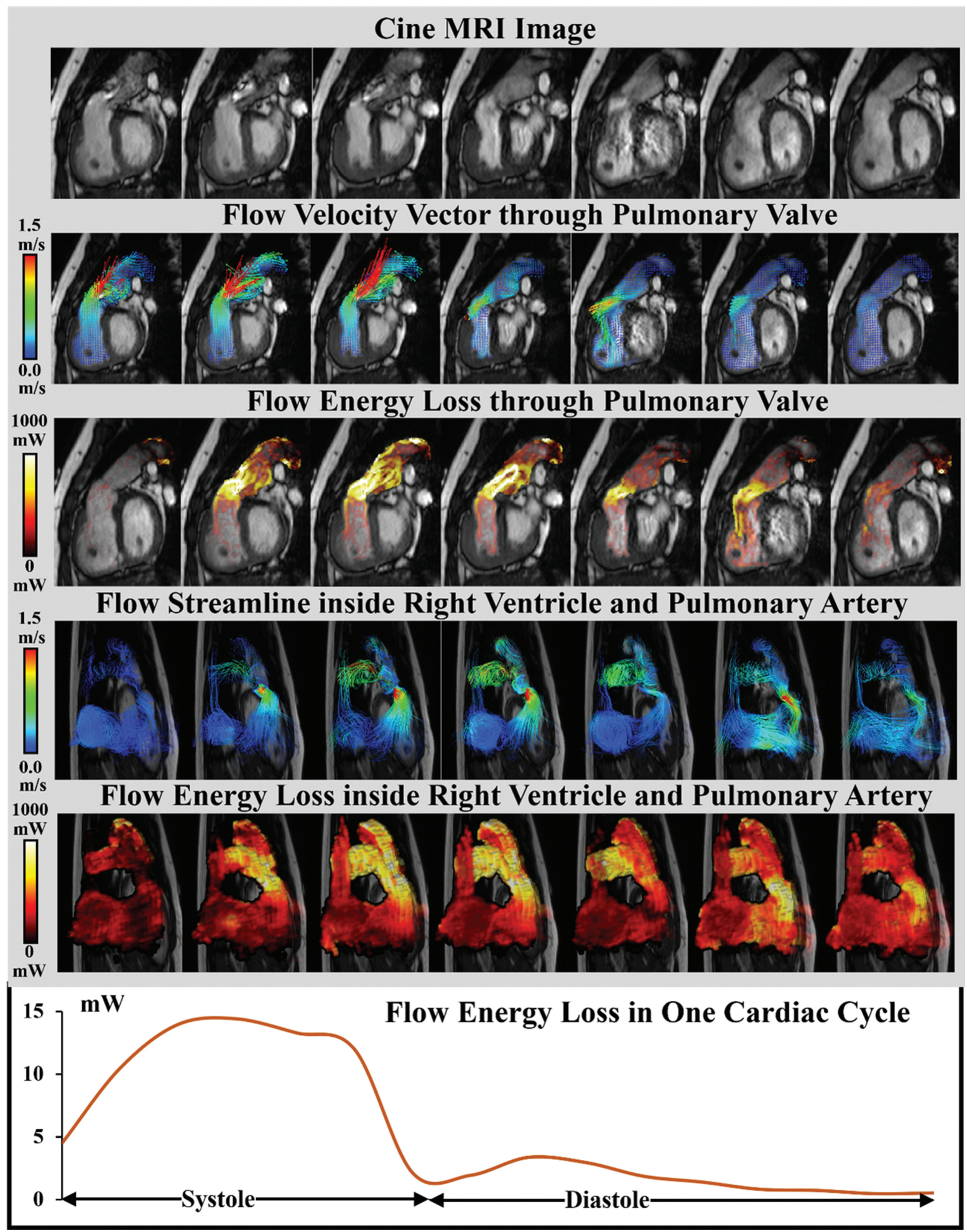

Fig. 6 EL example in one cardiac cycle in a pulmonary valve disease case. This case represents PSR. EL is high in the pulmonary artery caused after the stenotic jet and flow detachment during the systole due to the afterload of the valve stenosis, and is also high in the RV and RVOT during the diastole due to the preload caused by the regurgitation jet itself and by the flow collision of the regurgitation jet with tricuspid inflow. The upper three rows are the sagittal 2D view of the pulmonary valve (the first row), with flow velocity vector (the second row), and with 2D flow EL (the third row) in one cardiac cycle, whereas the lower 2 rows are 3D flow streamline (the fourth row) and 3D EL (fifth row) of 3D front view of the RV in one cardiac cycle (Unpublished data). EL, energy loss; PSR, pulmonary stenosis with regurgitation; $\mathrm{RV}$, right ventricle; RVOT, right ventricle outflow tract. 


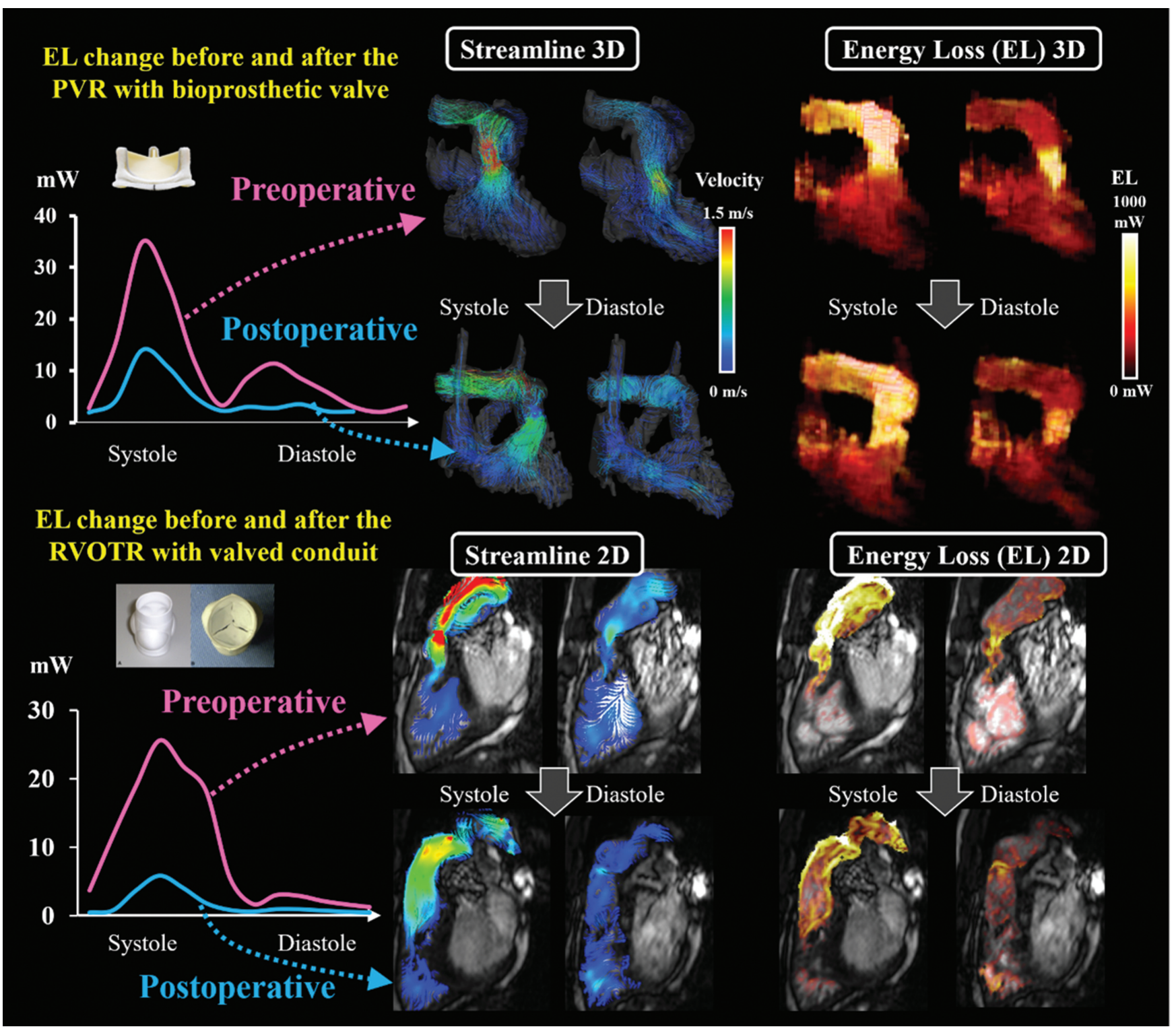

Fig. 7 EL and flow pattern change before and after the surgical intervention of pulmonary valve and RVOT with a bioprothetic PVR and with a valved conduit RVOTR (Unpublished data). EL, energy loss; PSR, pulmonary stenosis with regurgitation; RV, right ventricle; RVOT, right ventricle outflow tract.

physiology, with the increase in regurgitation volume, ELI increases with the decrease in $\mathrm{CI}$, irrespective of isolated PR $(n=17)$ or PSR $(n=26)$, and finally drops in the RV failure state, where both ELI and CI are small, but the $\mathrm{EL} / \mathrm{CO}$ ratio is higher than the normal RV (Fig. 9c). This plot is consistent with a previous study (Fig. 9a) (1) $^{1}$ that describes the concept of EL as a parameter of the energy generated by the ventricle. From the observed data, the surgical index of the pulmonary valve disease should include the EL index and the EL/CO ratio, whose cutoff values were 2.37 and $0.90 \mathrm{~mW} \cdot \mathrm{min} / \mathrm{L}$, respectively (AUC: 0.83 and 0.93 , respectively), predicting the classical surgical indications in the guideline (Fig. 9a). ${ }^{80}$
Because EL in this definition includes a partial differential equation in space, it is dependent on the spatial resolution of $4 \mathrm{D}$ flow MRI. ${ }^{46}$ The measurement error in PC-MRI is easily amplified from the definition that includes the square of the differential. Thus, the filtering process of raw data should be refined before the measurement in 4D flow MRI.

As a representative parameter of cardiac workload, in addition to the pulmonary valve disease sample demonstrated here, a significant amount of clinical evidence has been reported, suggesting that EL correlates with the severity of diseases and can predict the prognosis of the cardiac function. The preload and afterload increase in aortic valve 


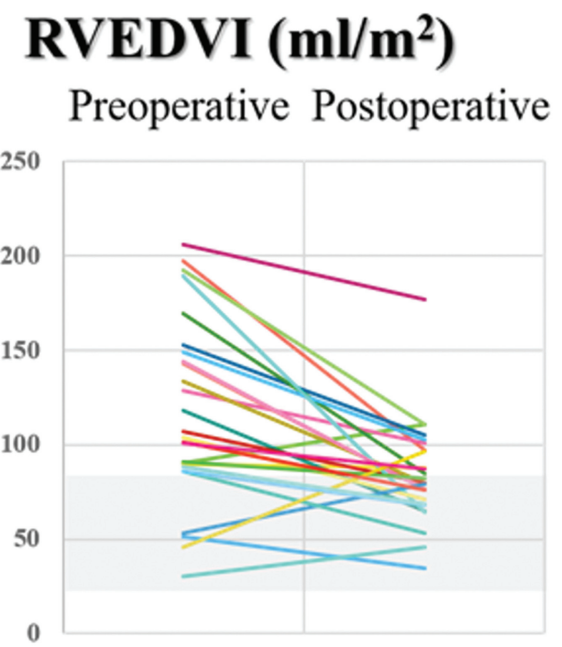

\section{$\operatorname{RVESVI~}\left(\mathbf{m l} / \mathbf{m}^{2}\right)$}
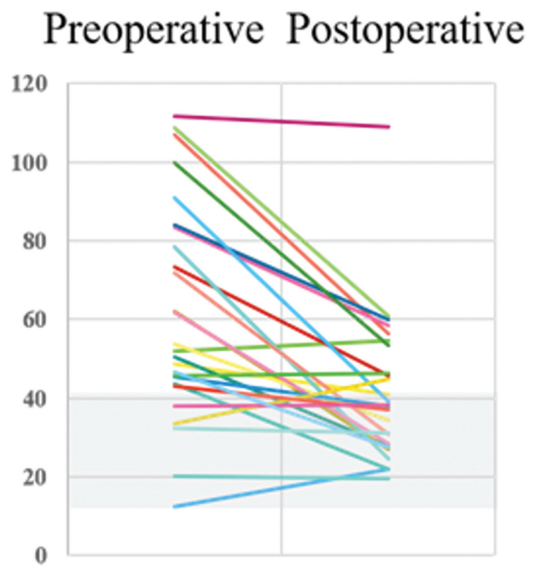

RVEF (\%)
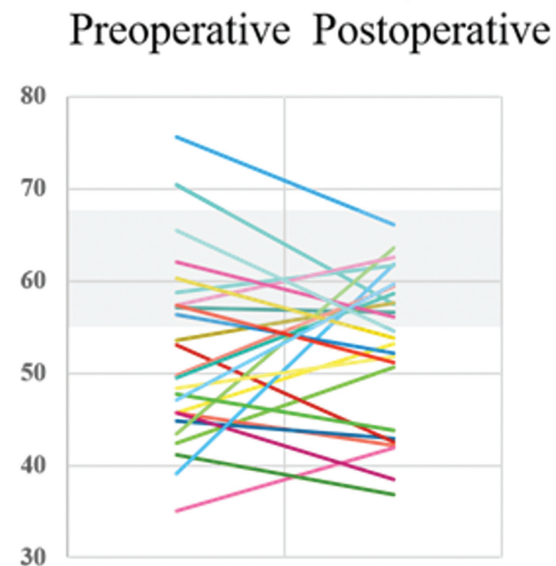

Fig. 8 RV reverse remodeling after the PVR and RVOTR with valved conduit. The shaded region represents the normal range of data of healthy volunteers. ${ }^{64}$ (reference 64 ). The cutoff values of the RV volume and function at which normalization could be expected were $149.08 \mathrm{ml} / \mathrm{m} 2,71.73 \mathrm{ml} / \mathrm{m}^{2}$, and $47.1 \%$ for RVEDV, RVESV, and RVEF, respectively, and the corresponding AUC $0.840,0.931$, and 0.921, respectively (unpublished data). AUC, corresponding area under the curve; PVR, pulmonary valve replacement; RV, Right ventricular; RVOTR, right ventricle outflow tract reconstruction.

disease has been reported to increase EL. ${ }^{54,58}$ Recovery from the heart failure state after resynchronization therapy ${ }^{59}$ or with medication therapy ${ }^{63}$ successfully reduces EL with the optimization of vortex flow duration ${ }^{59}$ and size. ${ }^{63}$ EL has also been reported to predict the postoperative prognosis of ventricular function, based on the observation of left ventricular remodeling $^{26}$ after AVR, and RV deterioration ${ }^{57}$ after the tetralogy of Fallot repair. In these diseased state with increased workload, EL in the systemic and pulmonary circulation measured with 4D flow MRI or EL in the LV chamber measured with echocardiography VFM was prominently high or apparently abnormal compared with the reference values and their standard deviations (SDs) of 4D flow MRI $^{64}$ and of echocardiography VFM. ${ }^{55,60}$ These facts suggest the reproducibility and feasibility of EL as a parameter to estimate cardiac workload in 4D flow MRI or in echocardiography VFM.

\section{Parameter to Evaluate Kinetic Energy of Turbulence}

Rather than parameters defined with a partial differential in space or time, which always have a problem of temporal or spatial resolution, a novel parameter named "turbulent kinetic energy (TKE)," based on a completely different concept to evaluate the kinetic energy of turbulent flow based on the intravoxel ensemble difference, has been derived and applied to cardiac diseases particularly those that cause a turbulent jet flow. ${ }^{85}$ Based on the principle of PC-MRI, the bipolar velocity encoding gradient has a first gradient moment of $\mathrm{M}$ defined by the following formula:

$$
M_{1}=\int_{0}^{T} t G(t) d t
$$

where $G$ is the gradient strength and $T$ is the time of application. SD of intravoxel velocity, the intravoxel standard deviation (IVSD) acquired using different gradient moments, can be derived based on the analytical expression for a voxel signal under a first gradient moment. ${ }^{86}$ The PC-MRI signal under the first gradient moment $M_{l}$ is expressed as:

$$
S\left(k_{u}\right)=C \int_{-\infty}^{\infty} s(u) e^{-i k_{u} u} d u
$$

where $\mathrm{C}$ is a scaling factor influenced by the relaxation parameters, spin density, and receiver gain. Function $s(u)$ is the spin velocity distribution of the voxel; and $k_{u}=\gamma M_{l}$, where $\gamma$ is the gyromagnetic ratio. When the spins within a voxel are assumed to have a Gaussian velocity distribution, $s(u)$ can be expressed as:

$$
s(u)=\frac{1}{\sigma \sqrt{2 \pi}} \exp \left(-\frac{\left(u-u_{m}\right)^{2}}{2 \sigma^{2}}\right)
$$

where $\sigma$ is the $\mathrm{SD}$, and $u_{m}$ is the mean velocity. From equation (33), the PC-MRI signal is integrated as: 
K. Itatani et al.
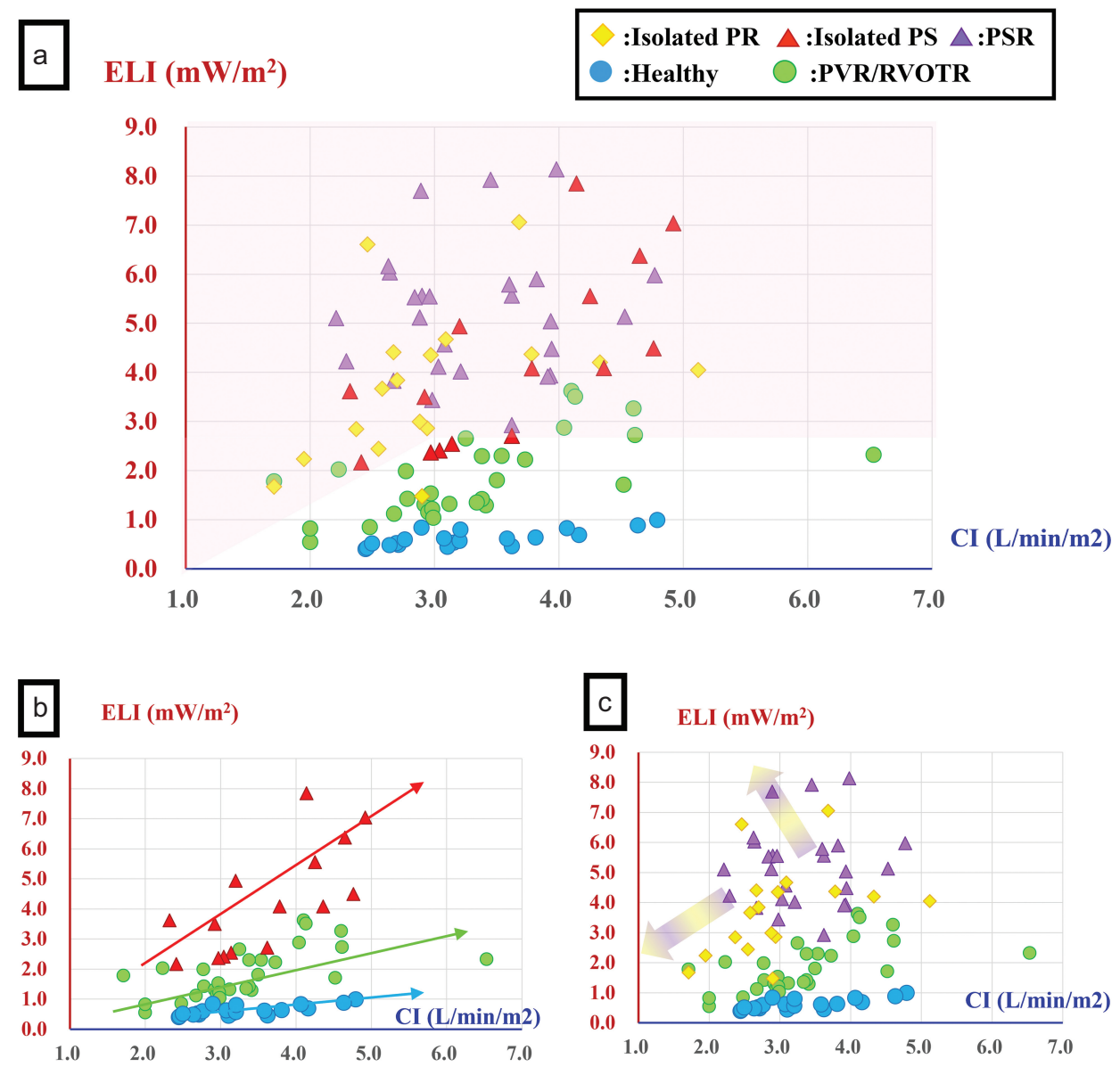

Fig. 9 Plot of EL index versus $\mathrm{Cl}$ and suggested surgical indication based on EL in pulmonary valve disease., measured with 4D flow MRI in ACHD pulmonary valve disease patients, including cases after the tetralogy of Fallot repair $(n=26)$, after the repair of PA/VSD $(n=7)$, after repair of DORV with PS $(n=3)$, after Ross or Ross-Konno procedure $(n=9)$, after repair of TGA $(n=4)$ and congenitally corrected TGA $(n=2)$, after repair of TCRV with VSD $(n=2)$, and others $(n=3)$ including congenital PS, congenital PR, and repeated RVOTR for residual shunt closure of Takeuchi root reconstructed for the Bland White Garland syndrome, in addition to 20 healthy volunteers. Among them, 26 postoperative patients were also evaluated in 4D flow MRI and, in these postoperative cases, 12 were cases with bioprosthetic pulmonary valve replacement (PVR) with bovine pericardial patch enlargement of ROVT, and 14 were cases with RVOTR with ePTFE tricuspid valved conduit with concomitant procedures. Among these 14 patients, there were cases that include tricuspid repair or replacement with bioprosthesis $(n=10)$, maze procedure or ventricular cryoablation $(n=5)$, aortic root or valve replacement $(n=4)$, and mitral plasty $(n=1)$. a: Plot of total cases. The EL/ $\mathrm{CO}$ ratio as well as the EL index itself, reflects the severity of the disease. b: Plot of the healthy, PS, and postoperative cases. The EL index increases with the increase of $\mathrm{Cl}$, but the slope or EL/CO ratio is significantly high in PS than those in healthy or postoperative states c: Plot of regurgitation. With the increase in regurgitation, $\mathrm{Cl}$ decreases but EL increases (unpublished data). ACHD, adult congenital heart disease; $\mathrm{Cl}$, cardiac index; $\mathrm{CO}$, cardiac output; DORV, double outlet right ventricle; ePTFE, expanded polytetrafluoroethylene; PA/VSD, pulmonary atresia with ventricular septal defect; PS, pulmonary stenosis; RVOTR, right ventricular outflow tract reconstruction; TCRV, two chamber right ventricle; TGA, transposition of the great arteries; VSD, ventricular septal defect.

$$
S\left(k_{u}\right)=C \exp \left(-\frac{\sigma^{2} k_{u}^{2}}{2}-i u u_{m} k_{u}\right)
$$

By combining the expressions of two different scan segments with different first gradient moments, i.e. $\left|k_{u 1}\right| \neq\left|k_{u 2}\right|$, SD $\sigma$ can be expressed using absolute values of the signals, ${ }^{86,87}$

$$
\sigma=\sqrt{\frac{2}{k_{u 1}^{2}-k_{u 2}^{2}} \ln \left(\frac{\left|S\left(k_{u 2}\right)\right|}{\left|S\left(k_{u 1}\right)\right|}\right)}
$$

In the derivation process of IVSD, magnitude images with different first gradient moments are used as signals, rather than phase images. 


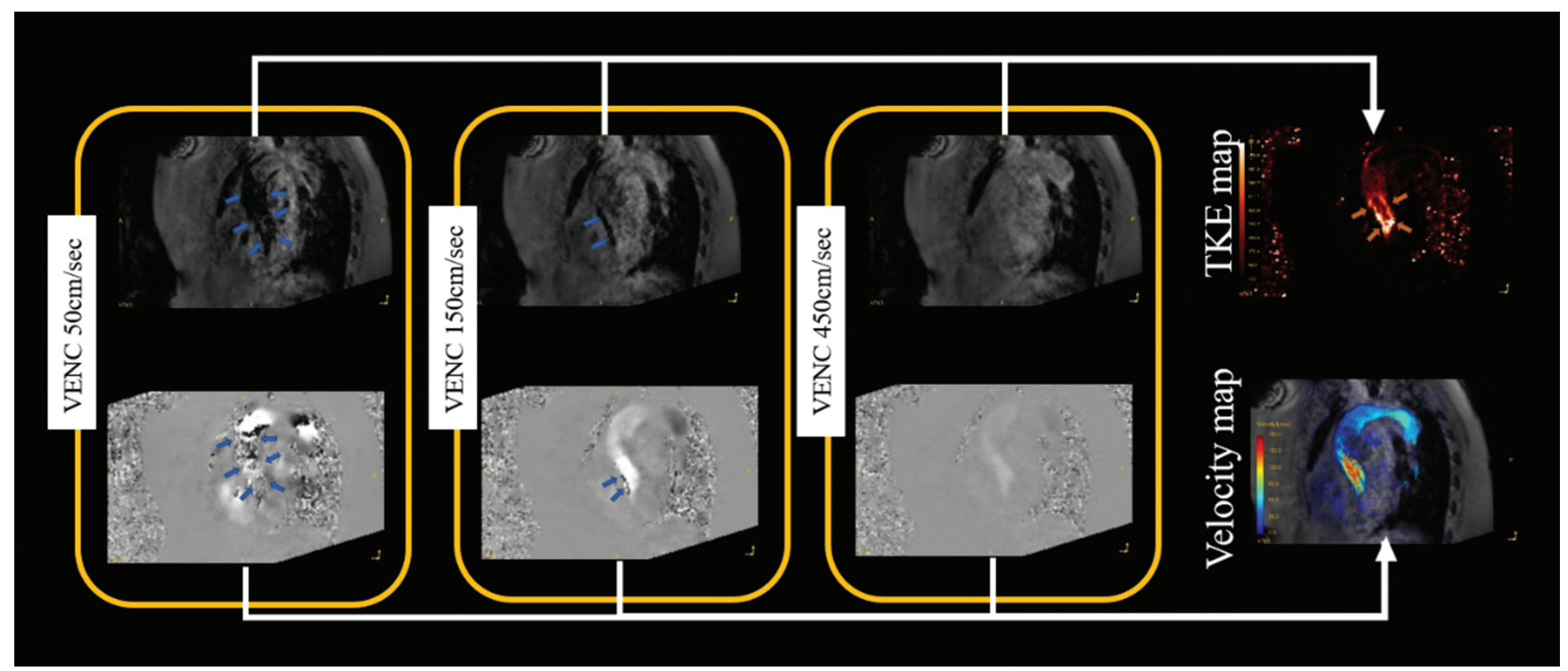

Fig. 10 Conceptual description of the method to create the TKE and velocity map simultaneously from the multiple-VENC scan. By combining scans with a moderate (VENC $150 \mathrm{~cm} / \mathrm{sec}$ ) and weak bipolar gradient (VENC $450 \mathrm{~cm} / \mathrm{sec)}$ ), and a reference scan without bipolar gradient (not shown), the turbulent kinetic energy is accurately calculated. In addition, by combining these scans, an accurate velocity map can be created (unpublished data). TKE, turbulent kinetic energy; VENC, velocity encoding.

Based on the theorem of turbulence, the measured flow velocity can be expressed as follows:

$$
u=\bar{u}+\hat{u}
$$

where $\bar{u}$ is the mean velocity averaged over time, space, or an ensemble, although in the ergodic theorem, these means should coincide with each other. $\hat{u}$ is the fluctuating velocity, whose mean value is 0 . From the definition of $\mathrm{SD}$, the root mean square of the fluctuating velocity in each direction $i$ should coincide with the measured IVSD of that direction.

$$
\sqrt{\overline{\hat{u}_{i}^{2}}}=\sigma_{i}
$$

In the Reynolds, the turbulence, or stress tensor, is defined as a second-order symmetrical tensor $\sigma \hat{\hat{u}}_{i} \hat{u}_{j}\left[\mathrm{~N} \mathrm{~m}^{-2}\right]$, and the diagonal elements are the Reynolds normal stresses in three directions. The Reynolds normal stresses add up to the tensor trace, which is an invariant of the Reynolds stress tensor and is thus insensitive to measurement directions. ${ }^{85}$ This tensor trace appears in the expression describing the average kinetic energy per unit volume $(\mathrm{K})$,

$$
K=\frac{1}{2} \rho \sum_{i} \overline{\bar{u}_{i}^{2}}+\frac{1}{2} \rho \sum_{i} \overline{\hat{u}_{i}^{2}}
$$

TKE is the second term of $K$, which is defined using the IVSD in three directions.

$$
T K E=\frac{1}{2} \rho \sum_{i} \overline{\hat{u}_{i}^{2}}=\frac{1}{2} \rho \sum_{i} \sigma_{i}^{2}
$$

The fluid dynamical concept of TKE is similar to that of the numerical turbulence calculation method of large eddy simulation (LES), in which subgrid scale fluctuation is assumed to be numerically negligible and filtered using operation such as Gaussian. ${ }^{87}$ Advantages of the TKE are the possibility of rescaling of the VENC with several scans at different first gradient moments, and the turbulent phenomena can be evaluated with low measurement error because of the filtering process with ensemble average. Independency of the spatial or temporal resolution without partial differential in space is another advantage of this parameter, enabling robustness in measurements.

The conceptual description of the TKE measurement in 4D flow MRI is illustrated in Fig. 10 (unpublished data). In this sample, a patient with hypertrophic cardiomyopathy, triple-VENC scans $(50,150$, and $450 \mathrm{~cm} / \mathrm{sec})$ were performed. The presence of jet blood flow in the LVOT caused a severe signal loss in the magnitude of the image under the influence of a strong bipolar gradient (VENC $50 \mathrm{~cm} / \mathrm{sec}$ ). The TKE is accurately calculated by combining the scans with a moderate bipolar gradient (VENC $150 \mathrm{~cm} / \mathrm{sec}$ ) and those with a weak bipolar gradient (VENC $450 \mathrm{~cm} / \mathrm{sec}$ ), along with a reference scan without a bipolar gradient (not shown). Furthermore, by combining these scans, an accurate velocity map can be created to facilitate the correction of over VENC aliasing in an accelerated jet flow. Although this triple-VENC scan takes 2.5 times longer than the standard 


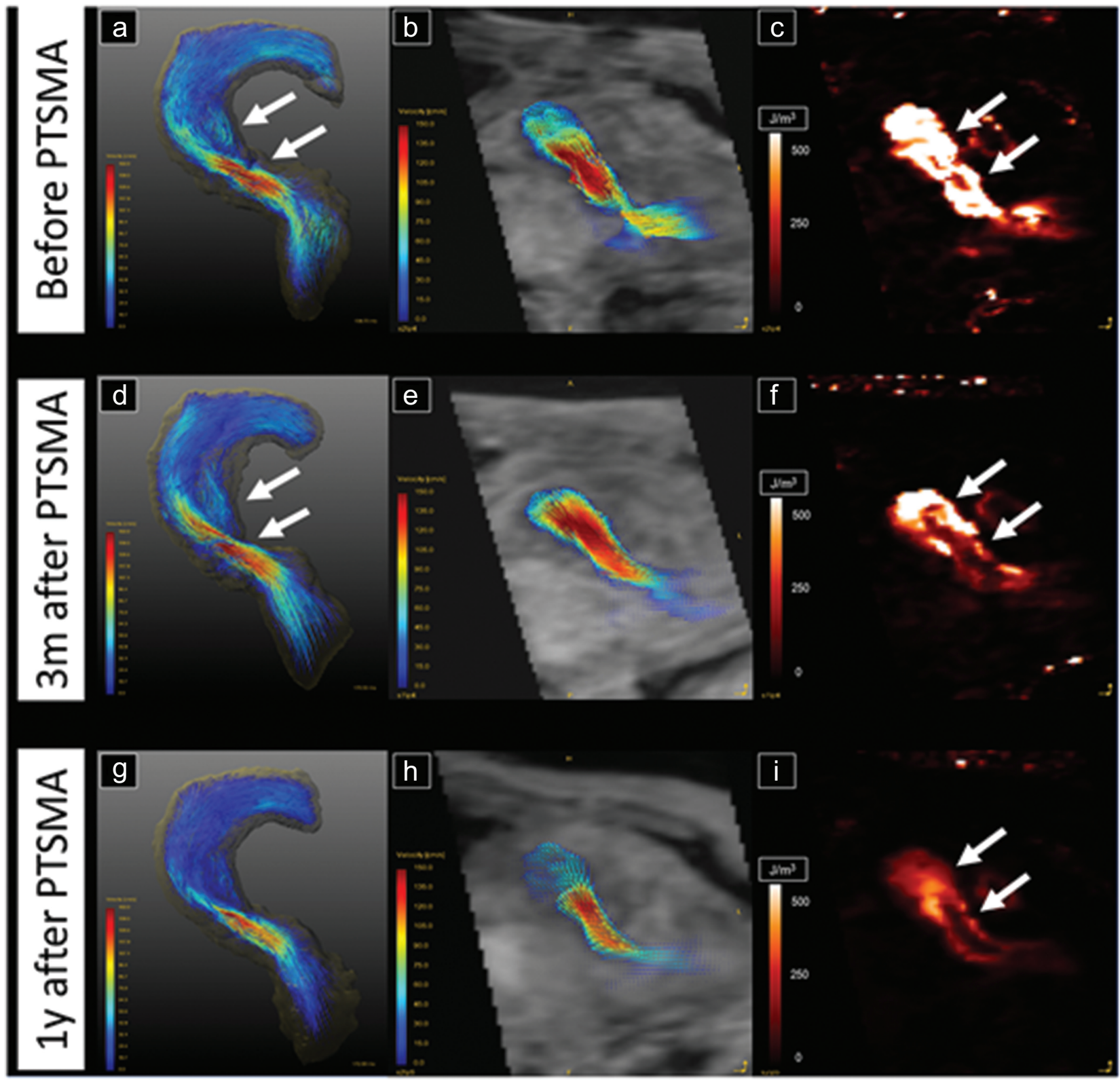

Fig. 11 TKE of a 79-year-old woman with HOCM before and after PTSMA. Prominent reduction of TKE was observed with released flow acceleration after PTSMA and reverse remodeling of the LV. ${ }^{95} \mathbf{a}-\mathbf{c}$ : Preoperative hemodynamics with streamline, local flow vector, and TKE, respectively. Before PTSMA, the LVOT PG measured on ultrasound was $51.0 \mathrm{mmHg}$. Cardiac MR showed severe cardiac hypertrophy (LV mass: $172.3 \mathrm{~g}$ ), and TKE was $21.02 \mathrm{~mJ}$. d-f: Hemodynamics 3 months after PSTMA with streamline, local flow vector, and TKE, respectively. Three months after PTSMA, the LVOT PG slightly decreased to $37.0 \mathrm{mmHg}$. No apparent changes in the flow pattern were observed in the streamline along with reduction in the TKE peak (15.27 mJ), although the LV mass did not shrink significantly (165.7 g). g-i: Hemodynamics 1 year after PSTMA with streamline, local flow vector, and TKE, respectively. ${ }^{95}$ One year after PTSMA, the LVOT PG was still slightly elevated at $43.0 \mathrm{mmHg}$. However, the flow pattern was improved with a decreased TKE peak of $8.74 \mathrm{~mJ}$. The left ventricular mass shrunk to $124.7 \mathrm{~g}$ and became NYHA functional Class I. HOCM, hypertrophic obstructive cardiomyopathy; LVOT, left ventricular outflow tract; PG, pressure gradient; PTSMA, percutaneous transluminal septal myocardial ablation; TKE, turbulent kinetic energy.

scan, the currently developed acceleration scans enable us to maintain the scan time within a clinically acceptable limit.

Despite having the unit of energy, the physiological meaning of energy is far from the cardiac work because this kinetic energy is not the kinetic energy of the blood flow averaged in the first term in equation (42) but is the fluctuating term of the total kinetic energy. Therefore, this is a parameter to estimate the extent of the turbulence, and not a parameter of the cardiac workload; moreover, the TKE is an absolutely different parameter when compared to the EL 
and related parameters. ${ }^{88,89}$ Because TKE does not depend on the spatial differential of the velocity fields such as EL or related parameters, TKE has sufficiently high accuracy theoretically ${ }^{12}$ and even in low spatial or temporal resolution in MRI measurements, according to the experimental validation study with optical PIV or PTV. ${ }^{14,16}$

Clinical application of the TKE is suitable for all types of heart diseases that have turbulent jet flow. A typical application is the AS, ${ }^{90}$ but the TKE is not an alternative for the pressure gradient in echocardiography, ${ }^{91}$ although post-stenotic pressure recovery has been examined with in vitro validation. ${ }^{92}$ Postoperative evaluation of AS has also been reported as one of the applications for TKE. ${ }^{93,94}$ In addition to valve stenosis jet, stenotic jet in left ventricular outflow obstruction in hypertrophic cardiomyopathy has also been reported. ${ }^{95}$ Regarding cardiomyopathy, energy dissipation in vortex flow in the dilated cardiomyopathy was also examined using $\mathrm{TKE}^{96}$ similar to EL. ${ }^{63}$ In addition, similar to applications in EL or viscous EL, the TKE has been applied to the CHDs including $\mathrm{RV}$ function in tetralogy of Fallot ${ }^{97}$ and coarctation of aorta. ${ }^{98}$ From the definition of TKE, highly disturbed flow with large ensemble SD, in which the typical flow study methods, such as echocardiography color Doppler, and PC-MRI are not always suitable, and those cases in which hemodynamics associated with jet flow are essential for the assessment of the disease would be good candidates for its application. Therefore, severity of the LVOTO or AS good is a candidate for this parameter. Fig. 11 illustrates the decrease in TKE after the treatment with the percutaneous transluminal septal myocardial ablation (PTSMA) to a hypertrophic obstructive cardiomyopathy (HOCM) case. ${ }^{95}$ The reduction of TKE in this case was followed by LV reverse remodeling with resolution of the symptom.

\section{Conclusion}

4D flow MRI is a widely used tool in cardiology and has the enabled comprehensive assessment of the cardiac function and hemodynamics in a wide range of cardiovascular diseases, including congenital heart disease, aortic disease, and heart valve diseases. In addition to the visualization of the flow streamline, the introduction of parameters to estimate the vortex, helical spiral, and turbulent flow has added numerous advantages to the 4D flow MRI in the determination and evaluation of therapeutic strategies, considering the improvement of the long-term prognosis of cardiac function after surgical procedures. The mathematical definitions of these parameters are sophisticated and provide a clear image of the unphysiological blood flow in each cardiovascular disease. Further refinement of these parameters with the progressive improvements in MRI machine specifications, temporal and spatial resolution, and measurement error could be expected.

\section{Funding}

Research works are funded by AMED (Japan Agency for Medical Research and Development) grant and Grant-inAid for Scientific Research from Japan Society for the Promotion of Science (KAKEN).

\section{Conflicts of Interest}

Keiichi Itatani is a founder of Cardio Flow Design Inc. and has a stock option of that company.

Shohei Miyazaki is CTO (Chief Technical Officer), an employee of Cardio Flow Design Inc., without stock option. The other authors have no conflict of interests.

\section{References}

1. Itatani K. Advances in Hemodynamic Research. Hauppauge: Nova Science Publisher, 2015.

2. Itatani K, Miyazaki S, Furusawa $\mathrm{T}$, et al. New imaging tools in cardiovascular medicine: computational fluid dynamics and 4D flow MRI. Gen Thorac Cardiovasc Surg 2017; 65:611-621.

3. Hwang J, Saha A, Boo YC, et al. Oscillatory shear stress stimulates endothelial production of O2- from $\mathrm{p} 47 \mathrm{phox}-$ dependent $\mathrm{NAD}(\mathrm{P}) \mathrm{H}$ oxidases, leading to monocyte adhesion. J Biol Chem 2003; 278:47291-47298.

4. Richter Y, Edelman ER. Cardiology is flow. Circulation 2006; 113:2679-2682.

5. Fukumoto Y, Hiro T, Fujii T, et al. Localized elevation of shear stress is related to coronary plaque rupture: a 3-dimensional intravascular ultrasound study with in-vivo color mapping of shear stress distribution. J Am Coll Cardiol 2008; 51:645-650.

6. Chatzizisis YS, Jonas M, Coskun AU, et al. Prediction of the localization of high-risk coronary atherosclerotic plaques on the basis of low endothelial shear stress: an intravascular ultrasound and histopathology natural history study. Circulation 2008; 117:993-1002.

7. Kato N, Yamagishi M, Itatani K, et al. Effects of blood flow dynamics on autologous pericardial degeneration in reconstructed pulmonary arteries. Interact Cardiovasc Thorac Surg 2018; 26:293-300.

8. Peiffer V, Sherwin SJ, Weinberg PD. Does low and oscillatory wall shear stress correlate spatially with early atherosclerosis? A systematic review. Cardiovasc Res 2013; 99:242-250.

9. Davies PF, Civelek M, Fang Y, Fleming I. The atherosusceptible endothelium: endothelial phenotypes in complex haemodynamic shear stress regions in vivo. Cardiovasc Res 2013; 99:315-327.

10. Gimbrone MA, García-Cardeña G. Vascular endothelium, hemodynamics, and the pathobiology of atherosclerosis. Cardiovasc Pathol 2013; 22:9-15.

11. Zhou J, Li YS, Chien S. Shear stress-initiated signaling and its regulation of endothelial function. Arterioscler Thromb Vasc Biol 2014; 34:2191-2198.

12. Dyverfeldt $P$, Gårdhagen $R$, Sigfridsson A, Karlsson M, Ebbers T. On MRI turbulence quantification. Magn Reson Imaging 2009; 27:913-922. 
13. Miyazaki S, Itatani K, Furusawa T, et al. Validation of numerical simulation methods in aortic arch using 4D Flow MRI. Heart Vessels 2017; 32:1032-1044.

14. Knobloch V, Binter C, Gülan U, et al. Mapping mean and fluctuating velocities by Bayesian multipoint MR velocity encoding-validation against 3D particle tracking velocimetry. Magn Reson Med 2014; 71:1405-1415.

15. Binter C, Gülan U, Holzner M, Kozerke S. On the accuracy of viscous and turbulent loss quantification in stenotic aortic flow using phase-contrast MRI. Magn Reson Med 2016; 76:191-196.

16. Rutkowski DR, Medero R, Ruesink TA, et al. Modeling physiological flow in Fontan models with four dimensional flow magnetic resonance imaging, particle image velocimetry, and arterial spin labeling. J Biomech Eng 2019; 141:121004.

17. Kilner PJ, Yang GZ, Mohiaddin RH, Firmin DN, Longmore DB. Helical and retrograde secondary flow patterns in the aortic arch studied by three-directional magnetic resonance velocity mapping. Circulation 1993; 88:2235-2247.

18. Itatani $\mathrm{K}$, Okada $\mathrm{T}$, Uejima $\mathrm{T}$, et al. Intraventricular flow velocity vector visualization based on the continuity equation and measurements of vorticity and wall shear stress. Jpn J Appl Phys 2013; 52:07HF16.

19. Itatani K. When the blood flow becomes bright. Eur Heart J 2014; 35:747-752a.

20. Deplano V, Meyer C, Guivier-Curien C, Bertrand E. New insights into the understanding of flow dynamics in an in vitro model for abdominal aortic aneurysms. Med Eng Phys 2013; 35:800-809.

21. Dewhurst P, Coats L, Parikh JD, Hollingsworth KG, Gan L. The role of flow rotation in the adult right atrium: a $4 \mathrm{D}$ flow cardiovascular magnetic resonance study. Physiol Meas 2020; 41:035007.

22. Gupta A, Jayaram R, Chaterjee AG, Sadhukhan S, Samtaney R, Verma MK. Energy and enstrophy spectra and fluxes for the inertial-dissipation range of two-dimensional turbulence. Phys Rev E 2019; 100:053101.

23. Arnold VI, Khesin BA. Topological methods in hydrodynamics. New York:Springer-Verlag, 1998.

24. Lorenz R, Bock J, Barker AJ, et al. 4D flow magnetic resonance imaging in bicuspid aortic valve disease demonstrates altered distribution of aortic blood flow helicity. Magn Reson Med 2014; 71:1542-1553.

25. Garcia J, Barker AJ, Collins JD, Carr JC, Markl M. Volumetric quantification of absolute local normalized helicity in patients with bicuspid aortic valve and aortic dilatation. Magn Reson Med 2017; 78:689-701.

26. Hohri Y, Itatani K, Numata S, et al. Blood flow energy loss: a predictor for the recovery of left ventricular function after bioprosthetic aortic valve replacement. Interact Cardiovasc Thorac Surg 2021; 33:339-347.

27. Takehara Y. 4D Flow when and how?. Radiol Med 2020; 125:838-850.

28. Sotelo J, Urbina J, Valverde I, et al. Three-dimensional quantification of vorticity and helicity from 3D cine PC-MRI using finite-element interpolations. Magn Reson Med 2018; 79:541553.

29. Schäfer M, Barker AJ, Kheyfets V, et al. Helicity and vorticity of pulmonary arterial flow in patients with pulmonary hypertension: Quantitative analysis of flow formations. J Am Heart Assoc 2017; 6:e007010.

30. Clough RE, Waltham M, Giese D, Taylor PR, Schaeffter T. A new imaging method for assessment of aortic dissection using four-dimensional phase contrast magnetic resonance imaging. J Vasc Surg 2012; 55:914-923.

31. Frydrychowicz A, Markl M, Hirtler D, et al. Aortic hemodynamics in patients with and without repair of aortic coarctation: in vivo analysis by $4 \mathrm{D}$ flow-sensitive magnetic resonance imaging. Invest Radiol 2011; 46:317-325.

32. Pike D, Shiu YT, Somarathna M, et al. High resolution hemodynamic profiling of murine arteriovenous fistula using magnetic resonance imaging and computational fluid dynamics. Theor Biol Med Model 2017; 14:5.

33. Cibis M, Bustamante M, Eriksson J, Carlhäll CJ, Ebbers T. Creating hemodynamic atlases of cardiac 4D flow MRI. J Magn Reson Imaging 2017; 46:1389-1399.

34. Callaghan FM, Bannon P, Barin E, et al. Age-related changes of shape and flow dynamics in healthy adult aortas: A 4D flow MRI study. J Magn Reson Imaging 2019; 49:90-100.

35. Shiina $Y$, Inai K, Miyazaki S, Nagao M. Aortic vorticity, helicity, and aortopathy in adult patients with tetralogy of fallot: Pilot study using four-dimensional flow magnetic resonance images. Pediatr Cardiol 2021; 42:169-177.

36. Kamada H, Ota H, Nakamura M, et al. Perioperative hemodynamic changes in the thoracic aorta in patients with aortic valve stenosis: A prospective serial $4 \mathrm{D}$-flow MRI study. Semin Thorac Cardiovasc Surg 2020; 32:25-34.

37. Garcia D, Pibarot P, Dumesnil JG, Sakr F, Durand LG. Assessment of aortic valve stenosis severity: A new index based on the energy loss concept. Circulation 2000; 101:765-771.

38. Bahlmann E, Gerdts E, Cramariuc D, et al. Prognostic value of energy loss index in asymptomatic aortic stenosis. Circulation 2013; 127:1149-1156.

39. Van Haesdock JM, Mertens L, Sizaire R, et al. Comparison by computerized numeric modeling of energy losses in different Fontan connections. Circulation. 1995;92:322-326.

40. de Leval MR, Dubini G, Migliavacca F, et al. Use of computational fluid dynamics in the design of surgical procedures: application to the study of competitive flows in cavo-pulmonary connections. J Thorac Cardiovasc Surg 1996; 111:502-513.

41. Dubini G, de Leval MR, Pietrabissa R, Montevecchi FM, Fumero R. A numerical fluid mechanical study of repaired congenital heart defects. Application to the total cavopulmonary connection. J Biomech 1996; 29:111-121.

42. Bove EL, de Leval MR, Migliavacca F, Guadagni G, Dubini G. Computational fluid dynamics in the evaluation of hemodynamic performance of cavopulmonary connections after the Norwood procedure for hypoplastic left heart syndrome. J Thorac Cardiovasc Surg 2003; 126:1040-1047.

43. Khunatorn Y, Mahalingam S, DeGroff CG, Shandas R. Influence of connection geometry and SVC-IVC flow rate ratio on flow structures within the total cavopulmonary connection: a numerical study. J Biomech Eng 2002; 124:364-77.

44. Hsia TY, Migliavacca F, Pittaccio S, et al. Computational fluid dynamic study of flow optimization in realistic models of the total cavopulmonary connections. J Surg Res 2004; 116:305-313. 
45. Itatani K, Miyaji K, Tomoyasu T, et al. Optimal conduit size of the extracardiac Fontan operation based on energy loss and flow stagnation. Ann Thorac Surg 2009; 88:565-572; discussion 572-573.

46. Grigioni M, Daniele C, Gaudio CD, et al. Numerical simulation of a realistic total cavo-pulmonary connection: Effect of unbalanced pulmonary resistances on hydrodynamic performance. Int J Arti Org 2003; 26:1005-1014.

47. Pekkan K, Kitajima HD, de Zelicourt D, et al. Total cavopulmonary connection flow with functional left pulmonary artery stenosis: angioplasty and fenestration in vitro. Circulation 2005; 112:3264-3271.

48. Itatani K, Miyaji K, Nakahata Y, Ohara K, Takamoto S, Ishii M. The lower limit of the pulmonary artery index for the extracardiac Fontan circulation. J Thorac Cardiovasc Surg 2011; 142:127-135.

49. de Zélicourt DA, Pekkan K, Parks J, Kanter K, Fogel M, Yoganathan AP. Flow study of an extracardiac connection with persistent left superior vena cava. J Thorac Cardiovasc Surg 2006; 131:785-791.

50. Whitehead KK, Pekkan K, Kitajima HD, Paridon SM, Yoganathan AP, Fogel MA. Nonlinear power loss during exercise in single-ventricle patients after the Fontan: insights from computational fluid dynamics. Circulation 2007; 116(11 Suppl):I165-I171.

51. Marsden AL, Vignon-Clementel IE, Chan FP, Feinstein JA, Taylor CA. Effects of exercise and respiration on hemodynamic efficiency in CFD simulations of the total cavopulmonary connection. Ann Biomed Eng 2007; 35:250-263.

52. Itatani K. Fluid dynamical considerations on the single ventricular physiology: Energetic optimization of the norwood and fontan procedures. PhD thesis, the University of Tokyo, 2011; 127094. (in Japanese).

53. Itatani $\mathrm{K}$, Ono $\mathrm{M}$, inventors; Minoru Ono, Keiichi ITATANI, assignee; University of Tokyo NUC. Blood flow visualizing diagnostic device. Publication number: WO2013077013A1. Application number: PCT/JP2012/063484.

54. Honda T, Itatani K, Miyaji K, Ishii M. Assessment of the vortex flow in the post-stenotic dilatation above the pulmonary valve stenosis in an infant using echocardiography vector flow mapping. Eur Heart J 2014; 35:306.

55. Hayashi $\mathrm{T}$, Itatani $\mathrm{K}$, Inuzuka $\mathrm{R}$, et al. Dissipative energy loss within the left ventricle detected by vector flow mapping in children: Normal values and effects of age and heart rate. J Cardiol 2015; 66:403-410.

56. Honda T, Itatani $\mathrm{K}$, Takanashi $\mathrm{M}$, et al. Exploring energy loss by vector flow mapping in children with ventricular septal defect: Pathophysiologic significance. Int J Cardiol 2017; 244:143-150.

57. Shibata $\mathrm{M}$, Itatani $\mathrm{K}$, Hayashi $\mathrm{T}$, et al. Flow energy loss as a predictive parameter for right ventricular deterioration caused by pulmonary regurgitation after tetralogy of fallot repair. Pediatr Cardiol 2018; 39:731-742.

58. Stugaard M, Koriyama H, Katsuki K, et al. Energy loss in the left ventricle obtained by vector flow mapping as a new quantitative measure of severity of aortic regurgitation: a combined experimental and clinical study. Eur Heart J Cardiovasc Imaging 2015; 16:723-730.

59. Kakizaki R, Nabeta T, Ishii S, et al. Cardiac resynchronization therapy reduces left ventricular energy loss. Int J Cardiol 2016; 221:546-548.
60. Akiyama K, Maeda S, Matsuyama T, et al. Vector flow mapping analysis of left ventricular energetic performance in healthy adult volunteers. BMC Cardiovasc Disord 2017; 17:21.

61. Akiyama K, Itatani K, Yamashita A, Sawa T. Visualization of suppressed intraventricular flow by constrictive pericarditis. J Clin Anesth 2017; 42:40-41.

62. Akiyama K, Naito Y, Kinoshita M, et al. Flow energy loss evaluation in a systolic anterior motion case after the ross pocedure. J Cardiothorac Vasc Anesth 2017; 31:2118-2122.

63. Nabeta T, Itatani K, Miyaji K, Ako J. Vortex flow energy loss reflects therapeutic effect in dilated cardiomyopathy. Eur Heart J 2015; 36:637.

64. Nakaji K, Itatani K, Tamaki N, et al. Assessment of biventricular hemodynamics and energy dynamics using lumentracking $4 \mathrm{D}$ flow MRI without contrast medium. J Cardiol 2021; 78:79-87.

65. Barker AJ, van Ooij P, Bandi K, et al. Viscous energy loss in the presence of abnormal aortic flow. Magn Reson Med 2014; 72:620-628.

66. Elbaz MSM, Scott MB, Barker AJ, et al. Four-dimensional virtual catheter: noninvasive assessment of intra-aortic hemodynamics in bicuspid aortic valve disease. Radiology 2019; 293:541-550.

67. Kamphuis VP, Roest AAW, van den Boogaard PJ, et al. Hemodynamic interplay of vorticity, viscous energy loss, and kinetic energy from 4D Flow MRI and link to cardiac function in healthy subjects and Fontan patients. Am J Physiol Heart Circ Physiol 2021; 320:H1687-H1698.

68. Rijnberg FM, van Assen HC, Hazekamp MG, Roest AAW. Tornado-like flow in the Fontan circulation: insights from quantification and visualization of viscous energy loss rate using 4D flow MRI. Eur Heart J 2019; 40:2170.

69. Han QJ, Witschey WR, Fang-Yen CM, et al. Altered right ventricular kinetic energy work density and viscous energy dissipation in patients with pulmonary arterial hypertension: A pilot study using 4D flow MRI. PLoS One 2015; 10:e0138365.

70. Nguyen YN, Tay ELW, Kabinejadian F, Ong CW, Ismail M, Leo HL. Ventricular vortex loss analysis due to various tricuspid valve repair techniques: an ex vivo study. Am J Physiol Heart Circ Physiol 2019; 317:H1312-H1327.

71. Burris NS, Hope MD. 4D flow MRI applications for aortic disease. Magn Reson Imaging Clin N Am 2015; 23:15-23.

72. Schäfer M, Barker AJ, Jaggers J, et al. Abnormal aortic flow conduction is associated with increased viscous energy loss in patients with repaired tetralogy of Fallot. Eur J Cardiothorac Surg 2020; 57:588-595.

73. Kamphuis VP, Westenberg JJM, van der Palen RLF, et al. Scan-rescan reproducibility of diastolic left ventricular kinetic energy, viscous energy loss and vorticity assessment using 4D flow MRI: analysis in healthy subjects. Int J Cardiovasc Imaging 2018; 34:905-920.

74. Elbaz MS, van der Geest RJ, Calkoen EE, et al. Assessment of viscous energy loss and the association with three-dimensional vortex ring formation in left ventricular inflow: In vivo evaluation using four-dimensional flow MRI. Magn Reson Med 2017; 77:794-805.

75. Schäfer M, Di Maria MV, Jaggers J, et al. High-degree Norwood neoaortic tapering is associated with abnormal flow conduction and elevated flow-mediated energy loss. J Thorac Cardiovasc Surg 2021; 162:1791-1804. 
76. Itatani K, Miyaji K, Qian Y, et al. Influence of surgical arch reconstruction methods on single ventricle workload in the Norwood procedure. J Thorac Cardiovasc Surg 2012; 144:130-138.

77. Asada S, Yamagishi M, Itatani K, Yaku H. Chimney reconstruction of the aortic arch in the Norwood procedure. J Thorac Cardiovasc Surg 2017; 154:e51-e54.

78. Miyazaki S, Miyaji K, Itatani K, et al. Surgical strategy for aortic arch reconstruction after the Norwood procedure based on numerical flow analysis. Interact Cardiovasc Thorac Surg 2018; 26:460-467.

79. Asada S, Yamagishi M, Itatani K, et al. Early outcomes and computational fluid dynamic analyses of chimney reconstruction in the Norwood procedure. Interact Cardiovasc Thorac Surg 2019; ivz040.

80. Stout KK, Daniels CJ, Aboulhosn JA, et al. 2018 AHA/ACC Guideline for the Management of Adults With Congenital Heart Disease: Executive Summary: A Report of the American College of Cardiology/American Heart Association Task Force on Clinical Practice Guidelines. J Am Coll Cardiol 2019; 73:1494-1563.

81. Therrien J, Provost Y, Merchant N, Williams W, Colman J, Webb G. Optimal timing for pulmonary valve replacement in adults after tetralogy of Fallot repair. Am J Cardiol 2005; 95:779-782.

82. Takigami M, Itatani K, Nakanishi N, et al. Evaluation using a four-dimensional imaging tool before and after pulmonary valve replacement in a patient with tetralogy of Fallot: a case report. J Med Case Rep 2019; 13:30.

83. Takigami M, Itatani K, Nakanishi N, et al. Reconstruction of right ventricular outflow tract stenosis and right ventricular failure after Ross procedure - comprehensive assessment of adult congenital heart disease with four-dimensional imaging: a case report. J Med Case Rep 2020; 14:113.

84. Yamagishi M. Right ventricular outflow reconstruction using a polytetrafluoroethylene conduit with bulging sinuses and tricuspid fan-shaped polytetrafluoroethylene valve. Oper Tech Thorac Cardiovasc Surg 2016; 21:211-229.

85. Dyverfeldt P, Kvitting JP, Sigfridsson A, Engvall J, Bolger AF, Ebbers T. Assessment of fluctuating velocities in disturbed cardiovascular blood flow: in vivo feasibility of generalized phase-contrast MRI. J Magn Reson Imaging 2008; 28:655-663.

86. Dyverfeldt P, Sigfridsson A, Kvitting JP, Ebbers T. Quantification of intravoxel velocity standard deviation and turbulence intensity by generalizing phase-contrast MRI. Magn Reson Med 2006; 56:850-858.
87. Chung TJ. Computational Fluid Dynamics, 2nd Edition. New York:Cambridge University Press, 2010; 706-713.

88. Iwata K, Sekine T, Tanaka I, Ando T, Orita E. Turbulent kinetic energy is different from viscous energy loss. Radiographics 2020; 40:2142-2144.

89. Dyverfeldt P, Bissell M, Barker AJ, et al. 4D flow cardiovascular magnetic resonance consensus statement. J Cardiovasc Magn Reson 2015; 17:72.

90. Dyverfeldt P, Hope MD, Tseng EE, Saloner D. Magnetic resonance measurement of turbulent kinetic energy for the estimation of irreversible pressure loss in aortic stenosis. JACC Cardiovasc Imaging 2013; 6:64-71.

91. Binter C, Gotschy A, Sündermann SH, et al. Turbulent kinetic energy assessed by multipoint 4-dimensional flow magnetic resonance imaging provides additional information relative to echocardiography for the determination of aortic stenosis severity. Circ Cardiovasc Imaging 2017; 10:

92. Ha H, Kim GB, Kweon J, et al. Turbulent kinetic energy measurement using phase contrast MRI for estimating the post-stenotic pressure drop: In vitro validation and clinical application. PLoS One 2016; 11:e0151540.

93. Giese D, Weiss K, Baeßler B, et al. In vitro evaluation of flow patterns and turbulent kinetic energy in trans-catheter aortic valve prostheses. MAGMA 2018; 31:165-172.

94. Yevtushenko P, Hellmeier F, Bruening J, et al. Surgical Aortic Valve Replacement: Are We Able to Improve Hemodynamic Outcome?. Biophys J 2019; 117:2324-2336.

95. Iwata K, Matsuda J, Imori Y, Sekine T, Takano H. Fourdimensional flow magnetic resonance imaging reveals the reduction in turbulent kinetic energy after percutaneous transluminal septal myocardial ablation in hypertrophic obstructive cardiomyopathy. Eur Heart J 2020; 41:1454.

96. Zajac J, Eriksson J, Dyverfeldt P, Bolger AF, Ebbers T, Carlhäll CJ. Turbulent kinetic energy in normal and myopathic left ventricles. J Magn Reson Imaging 2015; 41:1021-1029.

97. Fredriksson A, Trzebiatowska-Krzynska A, Dyverfeldt P, Engvall J, Ebbers T, Carlhäll CJ. Turbulent kinetic energy in the right ventricle: Potential MR marker for risk stratification of adults with repaired Tetralogy of Fallot. J Magn Reson Imaging 2018; 47:1043-1053.

98. Lantz J, Ebbers T, Engvall J, Karlsson M. Numerical and experimental assessment of turbulent kinetic energy in an aortic coarctation. J Biomech 2013; 46:1851-1858. 